\title{
The physics of single-side fluorination of graphene: DFT and DFT $+U$ studies
}

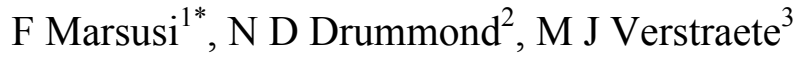 \\ ${ }^{1}$ Department of Energy Engineering and Physics, Amirkabir University of Technology, PO \\ Box 15875-4413, Tehran, Iran \\ ${ }^{2}$ Department of Physics, Lancaster University, Lancaster LA1 4YB, United Kingdom E-mail: \\ n.drummond@1ancaster.ac.uk \\ ${ }^{3}$ nanomat/QMAT/CESAM and European Theoretical Spectroscopy Facility, Université \\ de Liège, B-4000 Sart Tilman, Belgium E-mail: matthieu.verstraete@uliege.be
}

We present density functional theory (DFT) calculations of the electronic and magnetic properties of fluorine adatoms on a single side of a graphene monolayer. By extrapolating the results, the binding energy of a single fluorine adatom on graphene in the dilute limit is calculated. Our results confirm that the finite-size error in the binding energy scales inversely with the cube of the linear size of the simulation cell. We establish relationships between stability and $\mathrm{C}-\mathrm{F}$ bond nature, diffusion of fluorine adatoms and total magnetization in different configurations of adatoms. For single-side fluorination, $\mathrm{sp}^{2.33}$ is the maximum $\mathrm{p}$-content rehybridization found in the $\mathrm{C}-\mathrm{F}$ bond. We show that semilocal DFT cannot predict correctly the magnetic properties of fluorinated graphene and a higher level theory, such as DFT $+\mathrm{U}$ is needed. The results indicate a tendency of graphene to reduce the imbalance between adsorption on the two sublattices, and therefore total magnetization, through low-energy-barrier pathways on a time scale of $\sim 10 \mathrm{ps}$ at room temperature. The thermodynamically favored arrangements are those with the smallest total magnetization. Indeed, the electronic structure is intimately related to the magnetic properties and changes from semi-metallic to p-type half-metallic or semiconducting features, depending on the adatoms arrangement.

\section{Introduction}

\footnotetext{
1* Corresponding author. E-mail: marsusi@aut.ac.ir (Farah Marsusi)
} 
Functionalizing graphene with adatoms and admolecules is of significant interest in developing graphene-based electronics [1-2]. Doping can strongly perturb the electronic structure, leading to the formation of mid-gap states in semiconductors and the extreme modification of the optoelectronic and transport properties of graphene, which may be tuned through the type and concentration of dopant atoms. Electron or hole doping is achieved through charge-transfer and interaction with electron-donor molecules such as aniline and tetrathiafulvalene (n-type doping) and electron-acceptor molecules such as nitrobenzene, tetracyanoethylene, chlorosulfonic acid (p-type doping). These change the electronic structure and vibrational properties of graphene, as characterized by Raman shifts of the peak and width of the G band [3-6]. N-type graphene was also obtained by depositing CdSe quantum dots on the graphene surface [7]. Graphene doped substitutionally with boron and nitrogen displays $\mathrm{p}$ - and $\mathrm{n}$-type electronic properties, respectively [8-9]. On the other hand, halogen atoms ( $\mathrm{F}, \mathrm{Cl}, \mathrm{Br}$ and I), which have high electronegativity, are good candidates as external (as opposed to substitutional) dopants. The strength of carbonhalogen bonds decreases from fluorine to iodine, and the bond length increases. It is found that triiodide $\left(\mathrm{I}_{3}{ }^{-}\right)$and pentaiodide $\left(\mathrm{I}_{5}{ }^{-}\right)$are able to tune the electronic behavior of graphene from semimetallic to p-type metallic with increasing concentration [10-12]. Within the framework of the density functional theory, Tran et al. [13] have investigated the structure and electronic properties of halogenated graphene and found great differences between fluorine and other halogens: strong hybridization of $\mathrm{C}$ and $\mathrm{F}$ atoms leads to a buckled structure and significant change in $\pi$-bonding, and a distortion of the Dirac cone [13], while other halogen adatoms bind to $\mathrm{C}$ only through the $\mathrm{p}_{z}$ orbitals. Therefore, the planarity, Dirac cone and $\pi$-bonding are not destroyed by other halogen adatoms, which simply produce p-type metals [13]. Compared to other graphene derivatives, fluorinated graphene (FG) exhibits outstanding thermal and electronic properties [14]. The thermal stability of FG is even higher than that of pristine graphene [15]. The binding energy of fluorine on graphene is significantly larger than that of many other adatoms [16-17]. While this is essential as a measure of the stability of FG, many physical properties depend on the geometry and arrangement of multiple fluorine adatoms. Experimentally, there are two main methods to produce fluorographene [14]. In one method the fluorographene can be prepared by fluorinating existing graphene from one or both sides. In this approach, graphene is exposed to atomic $\mathrm{F}$ formed by decomposition of an appropriate fluorinating agent such as $\mathrm{XeF}_{2}, \mathrm{CF}_{4}$ or $\mathrm{SF}_{6}[15,18$, 19]. The degree of fluorinations is monitored by the Raman spectra. For fully fluorinated 
graphene all Raman features of pristine graphene disappear [15], which indicates significant changes are induced by fluorination. In the second method, FG is prepared by chemical or mechanical exfoliation of graphite fluoride [14, 20]. Synthesizing FG from the first approach is easier than extracting individual monolayers by mechanical exfoliation of graphite fluoride [15]. In addition to the two main approaches above, the successful selective patterning of single-sided $\mathrm{FG}\left(\mathrm{C}_{4} \mathrm{~F}\right)$ was reported [21] by laser irradiation of a fluoropolymer on graphene.

In this work, we present first-principles density functional theory (DFT) calculations to investigate the stability of single-side adsorption of fluorine on a graphene monolayer, against desorption or repulsion of adatoms. The single-side fluorination model can help us to understand the underlying physics of FG deposited on a substrate. As a first step, we inspect the stability of a single adatom on a graphene surface. To this end, we extrapolate our data to the infinitely dilute limit. Our leading finite-size error stems from the repulsive interactions between the image dipole moments along the $\mathrm{C}-\mathrm{F}$ bonds, and varies as $L^{-3}$, where $L$ is the linear size of the supercell.

Next, to capture the relationships between stability and nature of $\mathrm{C}-\mathrm{F}$ bonds, we perform calculations of the adsorption of fluorine adatoms, addimers, adtrimers and admolecules on a $5 \times 5$ supercell of graphene in different configurations. These models are representative of lowcoverage $\mathrm{FG}\left(\mathrm{CF}_{x}: x=n_{\mathrm{F}} / n_{\mathrm{C}}=0.02,0.04\right.$, and 0.06 , where $n_{\mathrm{F}}$ and $n_{\mathrm{C}}$ are the numbers of $\mathrm{F}$ adatoms and $\mathrm{C}$ atoms in the unit cell, respectively). The stability of fluorine adatoms on graphene has often been studied through the binding and formation energies [16, 22-26]. In our discussion we will also consider the effect of partial negative charges on fluorine adatoms. These may destroy the stability of small clusters of fluorine adatoms, by increasing atomic repulsion. To our knowledge this has not been considered in previous investigations. To investigate this point, we introduce another stability criterion: the energy $\Delta U_{B}$ required to separate two or more fluorine adatoms to an infinite distance from each other on the graphene surface. We find that, while a given configuration of adatoms may be stable based on its binding and formation energies, according to this new criterion, it may be unstable against repulsive interactions.

First-principles quantum mechanical methods have frequently been employed to investigate the stability, electronic and vibrational properties of FG [14, 27, 28]. However, limited efforts have been devoted to a detailed investigation of the $\mathrm{C}-\mathrm{F}$ bond nature at lower fluorine 
concentration. Here, we use the $\pi$-orbital axis vector (POAV) analysis, as described in Refs [29] and [30]. We present a discussion based on the relationship between local defects around the $\mathrm{C}-$ $\mathrm{F}$ bond and the stability of the structure, then we examine how $\mathrm{C}-\mathrm{F}$ chemical bonds behave at low density. Interestingly, POAV yields a central carbon rehybridization of $\mathrm{sp}^{2.33}$ for $\mathrm{CF}_{0.02}$ structure. This means the carbon atom is closer to $\mathrm{sp}^{3}$ hybridization than in a fullerene molecule, $\mathrm{C}_{60}$, with $\mathrm{sp}^{2.28}$ rehybridization under surface curvature [31-32]. In two-side fluorination, the covalency is almost fully $\mathrm{sp}^{3}$, but it increases less in single-sided highly FG.

The stability of FG is directly related to the total magnetic moment $\mathrm{M}$. For single hydrogen adatom on a graphene $5 \times 5$ unit cell, DFT reports agree on $M=1 \mu_{\mathrm{B}}$ [33-35], where $\mu_{\mathrm{B}}$ is Bohr magnetic moment. However, different DFT values for the magnetic moment of a fluorine adatom are reported in the literature, and the results are functional dependent. DFT within the generalized gradient approximation (GGA) predicts a nonmagnetic structure for a lower concentration of fluorine adatoms on graphene [24, 36-38]. Conversely, hybrid functionals predict $M=1 \mu_{\mathrm{B}}$ for the same concentration [39]. In this work, we apply DFT $+U$ to resolve this discrepancy. In agreement with hybrid functionals, $\mathrm{DFT}+\mathrm{U}$ shows that a single fluorine adatom induces a magnetic moment of $M=1 \mu_{\mathrm{B}}$ on graphene, and the magnetic properties have a close relationship with the stability of the structure. We will show that since graphene favors keeping the balance between two sublattices, spins are arranged with the lowest possible total magnetic moment, via exchange interactions. This finding is in agreement with the experimental observation that the measured number of paramagnetic centers is three orders of magnitude less than the number of fluorine adatoms in FG samples [40]. We show that partial charge transfer to the fluorine adatoms takes place, shifting the Fermi level of graphene into the valence cone. In addition, when the number of adatoms and their configuration destroys the balance between the two sublattices of graphene, spin up and down channels in the electronic structures are split. Therefore, the semi-metallic feature of graphene changes to a p-type half-metal in $\mathrm{CF}_{0.02}$, or a semiconductor in $\mathrm{CF}_{0.04}$ (intrinsic or p-type depending on the configuration) and $\mathrm{CF}_{0.06}$.

The information presented in this work will allow the further investigations of the bond nature, total magnetization, and thermodynamics of the fluorination process.

\section{Computational details}


The optimized geometries and binding energies of fluorine adatoms on graphene monolayers were obtained within the plane-wave pseudopotential DFT framework using the ABINIT code [41]. The cutoff energy on the plane-wave basis was set to $40 \mathrm{Ha}$. The PBE+U calculations were done within PAW spheres, while the convergence in PAW kinetic energy cutoff was found around $28 \mathrm{Ha}$. All atomic positions and in-plane lattice vectors were relaxed until the atomic forces were less than $6 \mathrm{meV} / \mathrm{A}^{\circ}$. A relatively large vacuum region of between 20-25 $\mathrm{A}^{\circ}$ along the $\mathrm{z}$-axis, was imposed to guarantee a vanishing interaction between the periodic images of the graphene layer.

To study the thermal stability of the $\mathrm{F}$ bonds to graphene, we performed molecular dynamics (MD) simulations using the LAMMPS program [42] and the reactive force-field ReaxFF [43], which can give a good description of bond breaking and bond formation during chemical reactions. The ReaxFF parameters for fluorographene were introduced in Refs. [44] and [45].

We then proceed to analyze the magnetic moment of each configuration in the $\mathrm{PBE}+\mathrm{U}$ framework [46] within PAW spheres, as implemented in the Quantum Espresso (QE) code [47], which allows the application of the repulsive $U$ in all space and not just in PAW spheres: this is more consistent for delocalized $\mathrm{p}$ orbitals in $\mathrm{C}$ or F. Norm-conserving Troullier-Martins pseudopotentials were used to represent the atomic cores [48]. Our criterion for the optimum value of parameter $U$ is the minimal one that restores the system of a single adatom absorbed on graphene to the expected magnetic moment near to $M=1 \mu_{\mathrm{B}}$. The partial occupancy of states was evaluated after a careful convergence test, with a small value of $0.001 \mathrm{eV}$ Gaussian smearing. After thorough scanning, we obtain the optimum value of $\mathrm{U}=2.5 \mathrm{eV}$.

For all DFT calculations we use the Perdew-Burke-Ernzerhof(PBE) [49] exchange-correlation functional. The binding energies of a single fluorine adatom were calculated in supercells of different size, to allow extrapolation of data to the infinite dilute limit. A $5 \times 5 \times 1$ Monkhorst-Pack k-point mesh was used to sample the Brillouin zone [50]. A finer sampling for smaller supercells was used to ensure the same k-point density was employed in all cases.

To study the stability of different FG structures, we calculated the binding energy and formation energy of different configurations within a $5 \times 5$ supercell. The average binding energy per adatom is defined by $\Delta E_{B}=\left[E(G)+n_{F} E(F)-E\left(G+n_{F} F\right)\right] / n_{F}$. Here $E(G)$ and $E(F)$ are 
the total energies of the graphene and fluorine ground states, respectively. $E\left(G+n_{F}\right)$ is the groundstate total energy of the graphene with $n_{F}$ fluorine adatoms. The formation energy per adatom, $\Delta E_{F}$, of $\mathrm{FG}$ relative to pristine graphene and the free $F_{2}$ molecule is an additional quantity that we use to characterize the stability of the configuration [22, 51]. $\Delta E_{F}$ is defined by $\left[E(G)+\frac{n_{F}}{2} E\left(F_{2}\right)-E\left(G+n_{F} F\right)\right] / n_{F}$, where $E\left(F_{2}\right)$ is the ground-state total energy of an isolated fluorine molecule.

We use another important stability criterion $\Delta U_{B}$ : the energy needed to separate fluorine adatoms to an infinite distance from each other on the graphene sheet, defined as $\Delta U_{B}=E(G)+$ $n_{F} E(F)-n_{F} \Delta E_{B_{\text {single }}}-E\left(G+n_{F} F\right)=n_{F}\left(\Delta E_{B}-\Delta E_{B_{\text {single }}}\right)$, where $\Delta E_{B_{\text {single }}}$ represents the binding energy of a single adatom on graphene. A negative value for $\Delta E_{B}$ or $\Delta E_{F}$ indicates that the fluorine adatoms on graphene are energetically unbound and will tend to leave the graphene surface. On the other hand, a negative $\Delta U_{B}$ indicates that the cluster of adatoms on the graphene surface would prefer to dissociate into isolated adatoms.

We have further compared the binding energy of $F_{2}$ admolecules on graphene in different configurations using $\Delta E_{B}=\left[E(G)+E\left(F_{2}\right)-E\left(G+F_{2}\right)\right]$, where $E\left(G+\mathrm{F}_{2}\right)$ represents the total energy of the admolecule on graphene. Van der Waals ( $\mathrm{vdW}$ ) interactions are required to describe the interaction between a molecule and a surface. Therefore, we apply the DFT-D2 nonlocal functional to modify the dispersion part of the PBE functional [52] in both relaxation and energy calculations including $\mathrm{F}_{2}$.

PBE activation energies for the diffusion of fluorine adatoms were calculated using the nudged elastic band (NEB) method [53], as implemented in the QE code. The numbers of successful thermally activated jumping processes per second is controlled by the energy barrier height $\Delta E_{h}$ from one site to a neighboring site. Due to the relatively large size of the cell, the NEB calculations were limited to five intermediate images between the initial and final structures of the path. The jumping rate to overcome the barrier height $\Delta E_{h}$ is $\Gamma=\Gamma_{0} \exp \left(\frac{-\Delta E_{h}}{k_{B} T}\right)$, where the $\Gamma_{0}$ is the ratio of the vibrational frequencies at the initial configuration to the frequencies at the saddle point. The parameters $\mathrm{k}_{\mathrm{B}}$ and $\mathrm{T}$ show the Boltzmann constant and temperature, respectively. All of our calculations were performed with spin-polarized wave functions unless otherwise stated. 


\section{Results and discussion}

\subsection{Binding energy of a single adatom in the infinitely dilute limit}

We have tried several different positions to adsorb F on graphene, as shown in Fig. 1(b). Our results show that in a $3 \times 3$ supercell the top position is more stable than the center and bridge positions by $0.49 \mathrm{eV}$ and $0.30 \mathrm{eV}$, respectively. The fluorine in the middle points will migrate spontaneously to the top position after relaxation. The top position, with a vertical C-F bond, with

a covalent bond of $\mathrm{sp}^{3}$ hybridization, is energetically the most favorable position, as previously reported for structures with a higher fluorine concentration $\left(\mathrm{C}_{2} \mathrm{~F}\right.$ and $\left.\mathrm{C}_{4} \mathrm{~F}\right)$ [16, 54]. However, one should notice that at higher concentration the interactions between fluorine atoms lead to $\mathrm{C}-\mathrm{F}$ bonds which are not perpendicular to the graphene plane. MD calculations using an NVT ensemble in a $7 \times 7$ graphene supercell with temperatures up to $400 \mathrm{~K}$ show that the fluorine atoms stay at the top position above a carbon atom even at high temperatures. The root-mean-square horizontal thermal fluctuations of the fluorine are less than $0.047 \mathrm{~A}^{\circ}$.

Before analyzing the parameters that affect the stability of FG, we extrapolate the binding energy of a fluorine adatom to the infinitely dilute limit. Computationally, the infinitely dilute limit can be described by a very large periodic supercell $(L \rightarrow \infty$, where $L$ is the linear size of the cell) to avoid interactions between periodic images of the adatom. Such calculations can be extremely expensive for sizes beyond a few hundred atoms. Therefore, we estimate $\Delta E_{B}$ by calculating the binding energy in a series of supercells with different sizes, and extrapolating the results to the infinite-size limit. We list in Table 1 the cell size and the spurious interactions between the periodic images (finite-size errors), which influence the binding energy. Our analysis shows that the interactions between periodic dipole moments induced by the fluorine adatom is the main origin of this error, and converges slowly with respect to system size, as seen in Table 1. Löwdin population analysis [55] confirms that the charge on $\mathrm{F}$ is mostly transferred from the central carbon atom beneath it, $\mathrm{C}(\mathrm{F})$ : see Fig. 1(c). This causes the defect to have a nonzero electric dipole moment. 
(a)
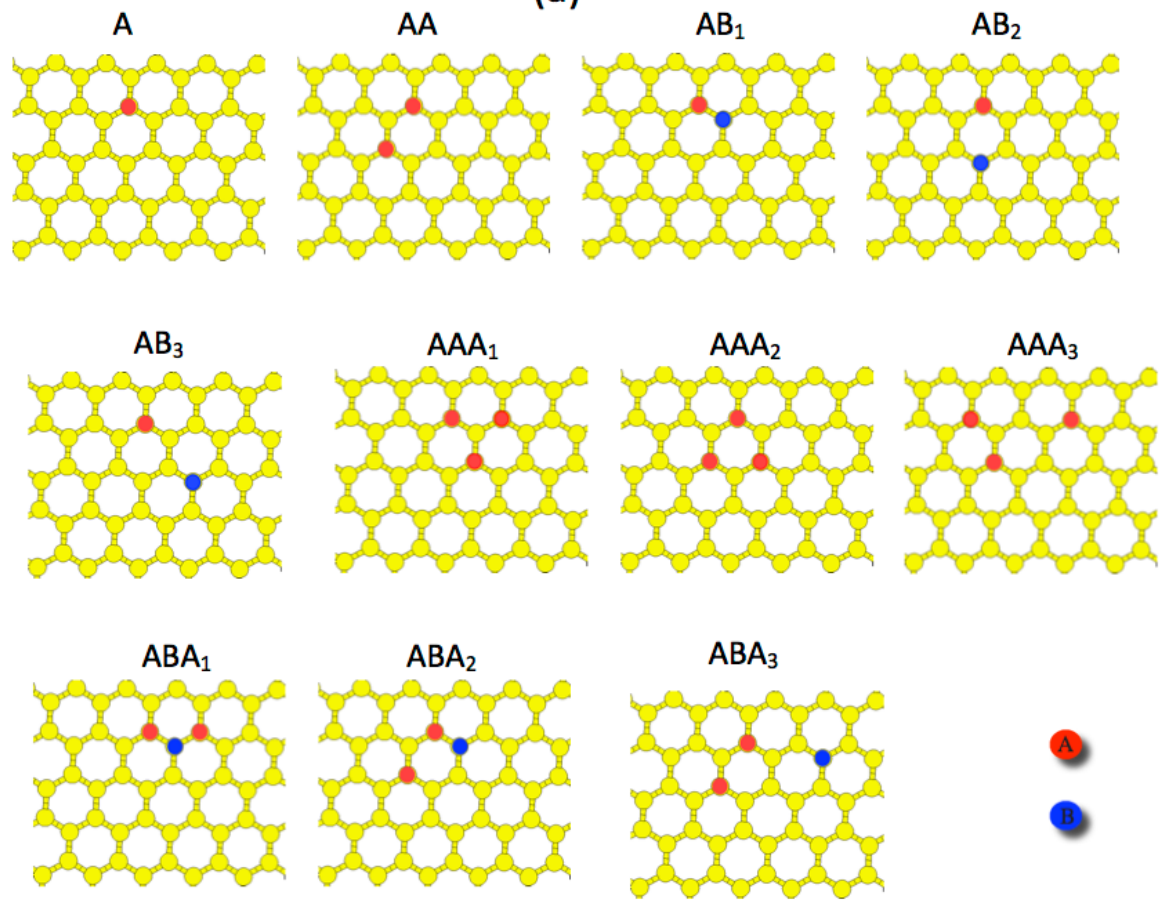

(b)

(c)
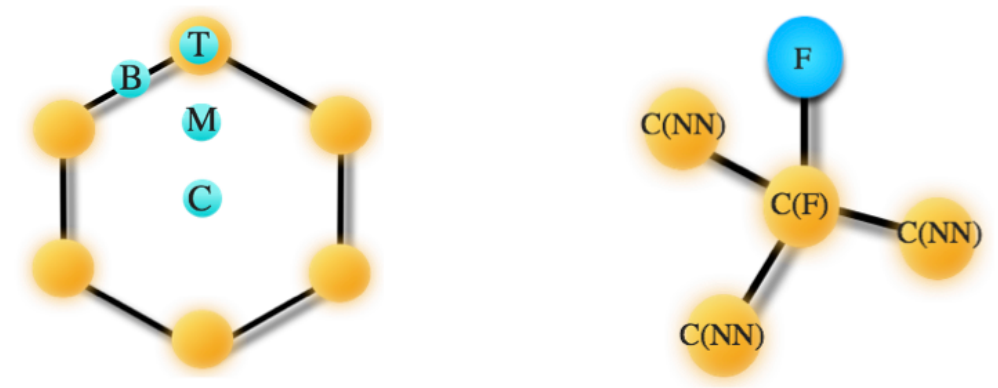

Fig. 1: (a): Different configurations for fluorine adatoms on a $5 \times 5$ supercell of graphene corresponding to $\mathrm{CF}_{0.02}, \mathrm{CF}_{0.04}$, and $\mathrm{CF}_{0.06}$. Adsorption sites of sublattices $\mathrm{A}$ and $\mathrm{B}$ are specified with red (A) and blue (B) circles, respectively. (b): Different possible positions are shown for a single adatom: top (T), bridge (B), middle (M), and center (C). (c): Representations of typical $\mathrm{C}(\mathrm{F})$ and $\mathrm{C}(\mathrm{NN})$ carbon atoms.

If one models the graphene layer crudely as a perfect metal then the interaction between fluorine adatoms (including the response of the graphene layer) can be calculated by the method of images. 
In this model, an isolated fluorine adatom above the free-standing graphene layer with a partial charge $q_{\mathrm{F}}$ is replaced by a charge $q_{\mathrm{F}}$ above the graphene layer and an image charge $-q_{\mathrm{F}}$

\begin{tabular}{cccccc}
\hline \hline Supercell & $\Delta E_{B}$ & $C(F)_{z}$ & $C(N N)_{z}$ & $C(F)_{Z} C(N N)_{z}$ & $d_{C-F}$ \\
& $(e V)$ & $\left(A^{\circ}\right)$ & $\left(A^{\circ}\right)$ & $\left(A^{\circ}\right)$ & $\left(A^{\circ}\right)$ \\
\hline $3 \times 3$ & 1.87 & 0.33 & 0.015 & 0.32 & 1.55 \\
$4 \times 4$ & 1.93 & 0.40 & 0.08 & 0.32 & 1.55 \\
$6 \times 6$ & 1.94 & 0.48 & 0.14 & 0.32 & 1.56 \\
\hline
\end{tabular}

Table 1. PBE predicted binding energy per adatom $\left(\Delta \mathrm{E}_{\mathrm{B}}\right) \cdot \mathrm{C}(\mathrm{F})_{\mathrm{z}}$ and $\mathrm{C}(\mathrm{NN})_{\mathrm{z}}$ show the vertical displacement of the carbon atom $\mathrm{C}(\mathrm{F})$ to which the fluorine adatom is bonded and the nearestneighbour carbon atoms $\mathrm{C}(\mathrm{NN})$ bonded to $\mathrm{C}(\mathrm{F})$, respectively after adatom adsorption from the initial graphene plane at $z=0 . C(F)_{z}-C(N N)_{z}$ is the vertical displacement of $\mathrm{C}(\mathrm{F})$ relative to the vertical displacement of its nearest neighbor $\mathrm{C}(\mathrm{NN}) . \mathrm{d}_{\mathrm{C}-\mathrm{F}}$ is the $\mathrm{C}(\mathrm{F})-\mathrm{F}$ bond length for a single fluorine adatom adsorbed on graphene. All energies are in $\mathrm{eV}$ and distances in $\mathrm{A}^{\circ}$.

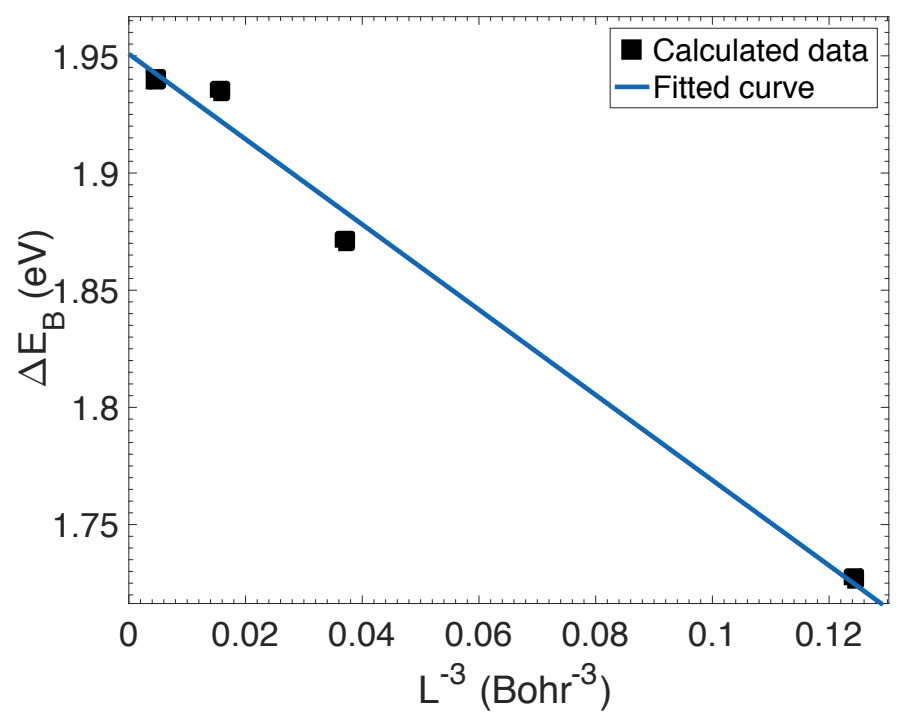

Fig. 2. (Color online) PBE binding energy of a single fluorine adatom on an $m \times m(m=3,4$ and 6) periodic supercell of graphene (black squares). The binding energy is extrapolated to the dilute limit of infinite cell size $(L \rightarrow \infty)$. The fitted parameter values are $\Delta E_{\mathrm{B}}(\infty)=1.95 \mathrm{eV}$ and $c=-1.82$ eV.Bohr ${ }^{3}$, with the root-mean-square error being $0.02 \mathrm{eV}$. 
on equal distance below the layer. This then gives an unscreened Coulomb dipole-dipole repulsion between fluorine adatoms at long range (but with the dipole moment being twice the naive value). The corresponding electrostatic energy of a 2D lattice of identical dipole moments is positive and falls off as $L^{-3}$ [56]. We investigated different finite-size extrapolation formulas and found the best-fit function, which gives the smallest root-mean-square error of $0.02 \mathrm{eV}$, reads as $\Delta E_{B}(L)=\Delta E_{B}(\infty)+c L^{-3}$ with $\mathrm{c}=-1.82 \mathrm{eV} \cdot B o h r^{3}$ : see Fig. 2 . Note that the positive error stemming from repulsive dipole-dipole contribution to the total energy, $E(G+F)$, results in " $c$ " being negative for the binding energy. Finally, the finite-size-corrected binding energy of a single fluorine adatom on a graphene surface is predicted to be $1.95 \mathrm{eV}$ by the PBE functional.

\subsection{Stability and nature of $C-F$ bonds.}

To reveal the underlying physics of adsorption of fluorine atoms on graphene, we have studied the stabilities of the different configurations shown in Fig. 1(a). Results from this work and Refs. [16] and [22] are listed in Table 2 and display a correlated relationship between the stability and $\mathrm{C}-\mathrm{F}$ bond nature. To understand this connection, we start with the adsorption of a single adatom on a $5 \times 5$ supercell of graphene monolayer which mimics the low concentration $\mathrm{CF}_{0.02}$.

Within PBE-PAW, the $\Delta E_{B}$ and $\Delta E_{F}$ of $\mathrm{CF}_{0.02}$ are predicted to be $1.85 \mathrm{eV}$ and $0.65 \mathrm{eV}$, respectively, much higher than room temperature $(\sim 26 \mathrm{meV})$. When compared with the same coverage of hydrogen adsorption on graphene, a clear distinction is observed. We obtain a negative formation energy of $-0.95 \mathrm{eV}$ for a single hydrogen adatom on graphene. The formation energy is a measure of stability against molecular desorption from the graphene surface. Therefore, in contrast to FG, hydrogenated graphene may readily dissociate into graphene and $\mathrm{H}_{2}$ molecules. This distinctive behavior is the result of the substantial difference in the $\mathrm{F}-\mathrm{F}$ and $\mathrm{H}-$ $\mathrm{H}$ bond energies. The experimental $\mathrm{F}-\mathrm{F}$ bond energy $(1.61 \mathrm{eV})$ is considerably smaller than the $\mathrm{C}-\mathrm{F}$ bond energy $(5.03 \mathrm{eV})$; in contrast, the $\mathrm{H}-\mathrm{H}$ bond energy $(4.48 \mathrm{eV})$ is larger than the value for $\mathrm{C}-\mathrm{H}(4.26 \mathrm{eV})$ [57]. 


\begin{tabular}{|c|c|c|c|c|c|}
\hline Configuration & $\begin{array}{l}\Delta E_{B} \\
(\mathrm{eV})\end{array}$ & $\begin{array}{c}\Delta U_{B} \\
(\mathrm{eV})\end{array}$ & $\begin{array}{l}\Delta E_{F} \\
(\mathrm{eV})\end{array}$ & $\begin{array}{c}M\left(\mu_{B}\right) \\
P B E\end{array}$ & $\begin{array}{c}M\left(\mu_{B}\right) \\
(P B E+U)\end{array}$ \\
\hline A $\quad\left(\mathrm{CF}_{0.02}\right)$ & 1.85 & & 0.65 & 0.00 & 1 \\
\hline $\mathrm{AA}\left(\mathrm{CF}_{0.04}\right)$ & 1.68 & -0.34 & 0.54 & 0.14 & 1.6 \\
\hline $\mathrm{AB}_{1}\left(\mathrm{CF}_{0.04}\right)$ & 2.00 & 0.30 & 0.86 & 0.00 & 0 \\
\hline $\mathrm{AB}_{2}\left(\mathrm{CF}_{0.04}\right)$ & 2.08 & 0.46 & 0.94 & 0.00 & \\
\hline $\mathrm{AB}_{3}\left(\mathrm{CF}_{0.04}\right)$ & 1.92 & 0.13 & 0.78 & 0.00 & \\
\hline $\mathrm{AAA}_{1}\left(\mathrm{CF}_{0.06}\right)$ & 1.53 & -0.94 & 0.40 & 1.00 & 3 \\
\hline $\mathrm{AAA}_{2}\left(\mathrm{CF}_{0.06}\right)$ & 1.64 & -0.62 & 0.51 & 1.00 & 3 \\
\hline $\mathrm{AAA}_{3}\left(\mathrm{CF}_{0.06}\right)$ & 1.64 & -0.64 & 0.51 & 0.50 & \\
\hline $\mathrm{ABA}_{1}\left(\mathrm{CF}_{0.06}\right)$ & 1.97 & 0.37 & 0.84 & 0.48 & 1 \\
\hline $\mathrm{ABA}_{2}\left(\mathrm{CF}_{0.06}\right)$ & 1.93 & 0.23 & 0.79 & 0.55 & \\
\hline $\mathrm{ABA}_{3}\left(\mathrm{CF}_{0.06}\right)$ & 1.77 & -0.25 & 0.63 & 0.18 & \\
\hline${ }^{\mathrm{a}} \mathrm{CF}_{1}$ (Chair) & 2.86 & & & & \\
\hline${ }^{\mathrm{b}} \mathrm{CF}_{0.25}$ & 3.00 & & & & \\
\hline
\end{tabular}

Table 2. PBE-PAW predicted binding energy $\left(\Delta \mathrm{E}_{\mathrm{B}}\right)$ and formation energy $\left(\Delta \mathrm{E}_{\mathrm{F}}\right)$ per adatom and $\Delta \mathrm{U}_{\mathrm{B}}$. The Calculated total magnetic moment $\mathrm{M}$ within PBE-PAW and PBE+U (for some configurations) are shown in $\mu_{B} .{ }^{a}$ Data are taken from Ref. [22]. ${ }^{b}$ Data are taken from Ref. [16].

Significant electronegativity differences between carbon and fluorine atoms lead to an ionic character for the $\mathrm{C}-\mathrm{F}$ bond. Two parameters determine the degree of ionic character in the $\mathrm{C}-\mathrm{F}$ bond: (i) the amount of charge transferred from graphene to fluorine and (ii) the local distortion about $\mathrm{C}(\mathrm{F})$ on graphene, which the corresponding parameters are introduced in Table 3. Löwdin analysis results show a significant charge of about $-0.38|\mathrm{e}|$, which is transferred mostly to the fluorine $\mathrm{p}_{z}$ orbital, accompanied by a charge depletion of $+0.27|e|$, mainly on the $\mathrm{p}_{z}$ orbital of the $\mathrm{C}(\mathrm{F})$ atom. In addition, $\mathrm{C}(\mathrm{F})$ puckers out of the plane by about $0.46 \mathrm{~A}^{\circ}$ and forms a local geometrical defect. A fully ionic bond would not disturb the planarity of graphene, since it involves predominantly the $\mathrm{p}_{z}$ orbital of $\mathrm{C}(\mathrm{F})$ atom, which is perpendicular to the graphene plane [58]. Hence, the calculated $0.46 \mathrm{~A}^{\circ}$ puckering of $\mathrm{C}(\mathrm{F})$ atom out of the plane is a sign of a (partly) covalent bond. Graphene has a strong $\mathrm{sp}^{2}$ network, and a strong oxidant like fluorine is needed to bond to it [58], leaving carbon in a new hybrid state. The calculated $\angle \mathrm{C}(\mathrm{NN}) \mathrm{C}(\mathrm{F}) \mathrm{F}$ and $\angle \mathrm{C}(\mathrm{NN}) \mathrm{C}(\mathrm{F}) \mathrm{C}(\mathrm{NN})$ angles $\left(\theta_{\mathrm{CCF}}\right.$ and $\left.\theta_{\mathrm{CCC}}\right)$ are $102.51^{\circ}$ and $115.45^{\circ}$, respectively, meaning hybridization falls between $\mathrm{sp}^{2}$ and $\mathrm{sp}^{3}$. The $\mathrm{C}-\mathrm{F}$ bond length is $1.57 \mathrm{~A}^{\circ}$ smaller than a typical ionic $\mathrm{C}-\mathrm{F}$ bond length of $3 \mathrm{~A}^{\circ}$, and larger than the typical covalent $\mathrm{C}-\mathrm{F}$ bond length of $1.43 \AA$ [59].

Likewise, the three $\mathrm{C}(\mathrm{NN})-\mathrm{C}(\mathrm{F}) \sigma$-bond lengths are $1.47 \mathrm{~A}^{\circ}$, larger than the corresponding values of $\mathrm{sp}^{2}$ bonds in graphene $\left(1.42 \mathrm{~A}^{\circ}\right)$, however smaller than $\mathrm{sp}^{3}$ bond lengths in diamond 
(1.54 $\AA$ ). We have performed MD calculations to study the effect of temperature on the C-F bond length. The mean value of the $\mathrm{C}-\mathrm{F}$ bond length predicted by MD calculations increases slightly from $1.48 \mathrm{~A}^{\circ}$ at $T=0 \mathrm{~K}$ to $1.50 \mathrm{~A}^{\circ}$ at $T=300 \mathrm{~K}$.

Now we inspect the degree of $p$ content in $\sigma$-orbitals by performing a POAV analysis, based on the orthogonality relationships between hybrid orbitals and the geometry of $\sigma$-bonds. From the mean values of the three $\theta_{\mathrm{CCF}}$ angles, POAV1 predicts $\mathrm{sp}^{2.33}$ hybridizations for the $\mathrm{C}-\mathrm{F}$ bond, which proves a semi-ionic character. At the same time, POAV2 predicts a deviation from $\mathrm{sp}^{2}$ into $\mathrm{sp}^{2.33}$ hybridization for the three $\sigma$-bonds between the $\mathrm{C}(\mathrm{F})$ central atom pointing towards the three carbon nearest neighbors $[\mathrm{C}(\mathrm{NN})]$. The binding energy of $1.85 \mathrm{eV}$ is relatively close to typical values for a semi-ionic $\mathrm{C}-\mathrm{F}$ bond strength $(2.08-2.38 \mathrm{eV})$. The typical covalent and ionic $\mathrm{C}-\mathrm{F}$ bond strengths are 2.67-2.88 eV and 1.08-1.56 eV, respectively [59]. Here it is noteworthy to compare these results with the bond nature of carbon in the most stable two-sided chair configuration of FG. We applied POAV analysis to the geometry parameters of the $\mathrm{CF}_{1}$ chair configuration, reported in Ref. [22] with a binding energy of $2.86 \mathrm{eV}$. Our results show the highly dominant covalent character of $\mathrm{C}-\mathrm{F}$ bonds with $\mathrm{sp}^{2.82}$ hybridization of carbon atoms corresponding to $108.1^{\circ}$ tetrahedral $\theta_{\mathrm{CCF}}$ angles and geometrical modification of the hexagonal $\theta_{\mathrm{CCC}}$ angles from $120^{\circ}$ to $110.8^{\circ}$.

Next, we consider adsorption of two adatoms on a $5 \times 5$ supercell, which models $\mathrm{CF}_{0.04}$. With two adatoms, different configurations are possible. Here we discuss the configurations shown in Fig. 1(a). First, we try a second adsorption on the next nearest neighbor site. We name this configuration AA, since the first and second adatoms are bonded to carbon atoms belonging to the same sublattice. The data in Table 3 show a slightly larger covalent nature for $\mathrm{C}-\mathrm{F}$ bonds, along with a smaller charge on fluorine, compared to adsorption of a single adatom. The mean values of $\theta_{\mathrm{CCF}}$ and $\theta_{\mathrm{CCC}}$ about each central carbon are slightly closer to the characteristic value of $\mathrm{sp}^{3}$ hybridization. In spite of the increasingly covalent nature, both the binding and formation energies are reduced, due to the repulsive interaction of the two fluorine atoms. Both energies are still positive and the system will not desorb the F atoms. However, we note that the negative charge on the two fluorine atoms may destroy the stability of the structure. We quantify the role of the repulsive interaction by calculating $\Delta U_{B}$, as described in Section 2, yielding a negative value of $-0.34 \mathrm{eV}$; see Table 2 . This indicates the AA configuration is not stable with respect to 
the repulsive interaction. Instead, when the second adatom adsorbs on the nearest neighbor site, belonging to the opposite sublattice $\left(\mathrm{AB}_{1}\right.$ arrangement in Fig.1(a)), all stability criteria are positive and the system is fully stable. Changing the position of the second adatom to the $\mathrm{AB}_{2}$ and $\mathrm{AB}_{3}$ configurations shown in Fig. 1(a), we have checked that all of these combinations are stable. Therefore, the stability depends on the symmetry of two adsorption sites. In the next section, we will see that the imbalanced binding of adatoms on the two graphene sublattices causes the unpaired electrons of the broken bonds to move across the graphene surface. Also, an imbalance between the two sublattices of graphene decreases the stability of the structure, since the number of broken $\pi$-bonds in the graphene network is equal to the number of defects minus the number of paired sites. As an example, in the AA and AB configurations two (2-0) and one (2-1) conjugated bonds are broken, respectively, making $\mathrm{AB}$ the more stable configuration. The absence of unpaired electrons in the $\mathrm{AB}$ arrangement makes the charge transfer to fluorine smaller, compared to the AA configuration. Therefore, the $\mathrm{C}-\mathrm{F}$ bonds in all of the $\mathrm{AB}$ configurations show less ionic character and more $\mathrm{p}_{\mathrm{z}}$-content hybridization $\left(\mathrm{sp}^{2.38}-\mathrm{sp}^{2.42}\right)$. The stability also depends on the position of the adatoms in an AB arrangement. Since the adatoms are further from each other, the geometrical distortion around the central carbon is smaller in the $\mathrm{AB}_{3}$ configuration, leading to less covalent nature for $\mathrm{C}-\mathrm{F}$ bonds, and a smaller binding energy, compared to $\mathrm{AB}_{1}$ and $\mathrm{AB}_{2}$. The two fluorine adatoms have a smaller separation in the $\mathrm{AB}_{1}$ configuration than in the $\mathrm{AB}_{2}$ configuration. The larger repulsive interaction does not allow each fluorine atom in the $\mathrm{AB}_{1}$ configuration to attract more charges than $0.28|\mathrm{e}|$. Because the adatoms are further from each other, in spite of attracting more significant charge, $\mathrm{AB}_{2}$ is a more stable configuration than $\mathrm{AB}_{1}$ and experiences a smaller repulsive energy, while the orientation of the $\mathrm{C}-\mathrm{F}$ bonds is closer to the geometry of $\mathrm{sp}^{3}$ structure. Table 3 reveals that changing the hybridization of $\mathrm{C}-\mathrm{F}$ results in $\mathrm{C}(\mathrm{F})$ puckering out of the graphene plane, and induces a local distortion about the $\mathrm{C}(\mathrm{F})$ in the $\sigma$-bond network. Therefore, the $\mathrm{C}-\mathrm{C}$ bond length increases from $1.42 \AA$ in pristine graphene towards $1.54 \mathrm{~A}^{\circ}$ in $\mathrm{sp}^{3}$ diamond. In the case of $\mathrm{AB}_{1}, \mathrm{~F}-\mathrm{F}$ repulsive interactions increase this phenomenon. The $\mathrm{F}-\mathrm{F}$ distance, even in $\mathrm{AB}_{1}$, is much larger than the $1.42 \AA$ interatomic bond length in $\mathrm{F}_{2}$ : the fluorine atoms prefer to bind covalently to carbon, instead of to nearby negatively charged fluorine atoms. 


\begin{tabular}{|c|c|c|c|c|c|c|c|c|c|c|}
\hline Configuration & $\begin{array}{c}d_{C-F} \\
A^{\circ}\end{array}$ & $\begin{array}{c}d_{C-C} \\
A^{\circ}\end{array}$ & $\begin{array}{l}d_{h} \\
A^{\circ}\end{array}$ & $\begin{array}{c}d_{F-F} \\
A^{\circ}\end{array}$ & $\overline{\theta_{C C F}}$ & $\begin{array}{c}\text { POAV1 } \\
s p^{x}\end{array}$ & $\overline{\theta_{C C C}}$ & $\begin{array}{c}P O A V 2 \\
s p^{x}\end{array}$ & $\begin{array}{l}\text { Charg } \\
C(F)\end{array}$ & $\begin{array}{c}\text { Charge } \\
F\end{array}$ \\
\hline A $\quad\left(\mathrm{CF}_{0.02}\right)$ & 1.57 & 1.47 & 0.46 & & 102.51 & 2.33 & 115.45 & 2.33 & +0.27 & -0.38 \\
\hline AA $\quad\left(\mathrm{CF}_{0.04}\right)$ & 1.55 & 1.47 & 0.50 & 2.93 & 103.02 & 2.36 & 115.07 & 2.37 & +0.27 & -0.35 \\
\hline $\mathrm{AB}_{1} \quad\left(\mathrm{CF}_{0.04}\right)$ & 1.45 & 1.57 & 0.68 & 2.37 & 103.38 & 2.38 & 114.79 & 2.40 & +0.30 & -0.28 \\
\hline $\mathrm{AB}_{2} \quad\left(\mathrm{CF}_{0.04}\right)$ & 1.49 & 1.50 & 0.50 & 3.07 & 103.88 & 2.42 & 114.44 & 2.41 & +0.30 & -0.31 \\
\hline $\mathrm{AB}_{3} \quad\left(\mathrm{CF}_{0.04}\right)$ & 1.50 & 1.49 & & & 103.27 & 2.38 & 114.88 & 2.38 & & \\
\hline $\mathrm{AAA}_{1}\left(\mathrm{CF}_{0.06}\right)$ & 1.53 & 1.47 & 0.55 & 2.99 & 103.09 & 2.36 & 115.01 & 2.37 & +0.27 & -0.33 \\
\hline $\mathrm{AAA}_{2}\left(\mathrm{CF}_{0.06}\right)$ & 1.52 & 1.48 & 0.60 & 2.98 & 103.72 & 2.41 & 114.54 & 2.41 & +0.28 & -0.33 \\
\hline $\mathrm{AAA}_{3}\left(\mathrm{CF}_{0.06}\right)$ & $\begin{array}{l}1.55 \\
1.55 \\
1.55\end{array}$ & $\begin{array}{l}1.47 \\
1.48 \\
1.48\end{array}$ & $\begin{array}{l}0.52 \\
0.50 \\
0.37\end{array}$ & $\begin{array}{l}2.95 \\
5.17 \\
4.46\end{array}$ & $\begin{array}{l}103.14 \\
103.19 \\
102.81\end{array}$ & $\begin{array}{l}2.37 \\
2.37 \\
2.35\end{array}$ & $\begin{array}{l}114.98 \\
114.60 \\
115.23\end{array}$ & $\begin{array}{l}2.37 \\
2.40 \\
2.35\end{array}$ & $\begin{array}{l}+0.27 \\
+0.28 \\
+0.28\end{array}$ & $\begin{array}{l}-0.34 \\
-0.33 \\
-0.35\end{array}$ \\
\hline $\mathrm{ABA}_{1}\left(\mathrm{CF}_{0.06}\right)$ & $\begin{array}{l}1.46 \\
1.42 \\
1.46\end{array}$ & $\begin{array}{l}1.58 \\
1.58 \\
1.58\end{array}$ & $\begin{array}{l}0.75 \\
0.88 \\
0.75\end{array}$ & $\begin{array}{l}2.37 \\
2.37 \\
3.40\end{array}$ & $\begin{array}{l}103.21 \\
102.95 \\
103.21\end{array}$ & $\begin{array}{l}2.37 \\
2.36 \\
2.37\end{array}$ & $\begin{array}{l}114.93 \\
115.13 \\
114.93\end{array}$ & $\begin{array}{l}2.43 \\
2.36 \\
2.43\end{array}$ & $\begin{array}{l}+0.30 \\
+0.29 \\
+0.30\end{array}$ & $\begin{array}{l}-0.28 \\
-0.24 \\
-0.28\end{array}$ \\
\hline $\mathrm{ABA}_{2}\left(\mathrm{CF}_{0.06}\right)$ & $\begin{array}{l}1.46 \\
1.44 \\
1.51\end{array}$ & $\begin{array}{l}1.58 \\
1.48 \\
1.50\end{array}$ & $\begin{array}{l}0.76 \\
0.72 \\
0.55\end{array}$ & $\begin{array}{l}2.39 \\
3.34 \\
2.95\end{array}$ & $\begin{array}{l}103.66 \\
103.55 \\
103.68\end{array}$ & $\begin{array}{l}2.40 \\
2.39 \\
2.40\end{array}$ & $\begin{array}{l}114.66 \\
114.51 \\
114.58\end{array}$ & $\begin{array}{l}2.41 \\
2.41 \\
2.40\end{array}$ & & \\
\hline $\mathrm{ABA}_{3}\left(\mathrm{CF}_{0.06}\right)$ & $\begin{array}{l}1.53 \\
1.51 \\
1.51\end{array}$ & $\begin{array}{l}1.49 \\
1.48 \\
1.49\end{array}$ & $\begin{array}{l}0.53 \\
0.41 \\
0.52\end{array}$ & $\begin{array}{l}3.92 \\
5.44 \\
2.94\end{array}$ & $\begin{array}{l}103.08 \\
102.72 \\
103.40\end{array}$ & $\begin{array}{l}2.36 \\
2.34 \\
2.38\end{array}$ & $\begin{array}{l}115.03 \\
115.30 \\
114.79\end{array}$ & $\begin{array}{l}2.37 \\
2.34 \\
2.39\end{array}$ & & \\
\hline${ }^{\mathrm{a}} \mathrm{CF}_{1}$ (Chair) & 1.37 & 1.58 & & & 108.1 & 2.82 & 110.8 & 2.82 & & \\
\hline${ }^{\mathrm{b}} \mathrm{C}_{4} \mathrm{~F}$ & 1.43 & 1.49 & & & 104 & 2.43 & 114 & 2.43 & & \\
\hline
\end{tabular}

Table 3. PBE-PAW predicted $\mathrm{C}(\mathrm{F})-\mathrm{F}\left(\mathrm{d}_{\mathrm{C}-\mathrm{F}}\right)$ and $\mathrm{C}(\mathrm{F})-\mathrm{C}(\mathrm{NN})$ bond lengths $\left(\mathrm{d}_{\mathrm{C}-\mathrm{C}}\right)$, puckering distance of $\mathrm{C}(\mathrm{F})$ atoms with respect to the initial position of graphene plane $\left(\mathrm{d}_{h}\right)$ in the $z$ direction and the distance between two fluorine adatoms $\left(\mathrm{d}_{\mathrm{F}-\mathrm{F}}\right)$ are listed. The mean value of $\theta_{\mathrm{CCF}}$ and the rehybridization $\left(\mathrm{sp}^{\mathrm{x}}\right)$ predicted by POAV1 for the carbon atom $\mathrm{C}(\mathrm{F})$ in the $\mathrm{C}-\mathrm{F}$ bond are also shown. $\theta_{\mathrm{CCC}}$ is the mean value of the internal hexagonal angle around $\mathrm{C}(\mathrm{F})$. Rehybridization $\left(\mathrm{sp}^{\mathrm{x}}\right)$ for the carbon atom $\mathrm{C}(\mathrm{F})$ in the $\mathrm{C}-\mathrm{C}$ bonds is predicted by POAV2. The charges on the $\mathrm{C}(\mathrm{F})$ and $\mathrm{F}$ atoms predicted by Löwdin analysis are given in units of electron charge $e$. All distances are in $\AA$ and angles in degrees. ${ }^{\mathrm{a}}$ Structural data are taken from Ref. [22]. ${ }^{\mathrm{b}}$ Structural data are taken from Ref. [16]

More configurations are possible with a third adatom, for which our $5 \times 5$ supercell models $\mathrm{CF}_{0.06}$. AAA and $\mathrm{ABA}$ configurations leave three and one broken $\pi$-bonds in the graphene 
network, respectively, which are not low-energy configurations for graphene. The result is that all AAA configurations shown in Fig. 1(a) are unstable with respect to $\Delta U_{B}$. For ABA configurations the situation is much better. In the more stable $\mathrm{ABA}_{1}$ and $\mathrm{ABA}_{2}$ mixed configurations the $\mathrm{C}-\mathrm{F}$ bond length becomes shorter, with slightly higher covalent nature. Due to repulsive interactions between partial charges on the central carbon atoms on opposite sites (nearest neighbors) $\mathrm{C}(\mathrm{F})-\mathrm{C}(\mathrm{NN})$, bond lengths increase and the configuration stabilizes. $\mathrm{ABA}_{1}$ is the most stable three-adatom structure among all mixed configurations, while $\mathrm{ABA}_{3}$ is not a stable structure, with a negative $\Delta U_{B}$. The stability criteria, including $\Delta U_{B}$, are comparable to those with two adatoms, in spite of the fact that the three adatoms are not distributed evenly in the mixed sublattices. Hence, one expects the stability and covalent nature to increase and the $\mathrm{C}-$ $\mathrm{F}$ bond lengths to decrease with the number of adatoms distributed evenly in the mixed sublattices. Contrary to two-side fluorination, full adsorption of $\mathrm{F}$ atoms over the same side of graphene plane $\left(\mathrm{CF}_{\mathrm{x}=1}\right)$ is not stable due to the repulsion between $\mathrm{F}$ atoms, and also topological frustration of the single side $\mathrm{sp}^{3}$ bonds, which should yield a curved surface. Among the highly concentrated single-side $\mathrm{FG}$ configurations, both $\mathrm{C}_{2} \mathrm{~F}\left(\mathrm{CF}_{0.5}\right), \mathrm{C}_{4} \mathrm{~F}\left(\mathrm{CF}_{0.25}\right)$ have been found previously to be stable, with $\mathrm{C}_{4} \mathrm{~F}$ being the most stable structure [16]. While the maximum attainable covalent nature can be $\mathrm{sp}^{2.82}$ for two-side full fluorination (FG chair structure), according to the geometries given in Ref. [16], it cannot exceed $\mathrm{sp}^{2.43}$ for single-side fluorination. $\mathrm{F}$ adatoms in the corresponding unit cell of $\mathrm{C}_{4} \mathrm{~F}$ are arranged in a configuration similar to $\mathrm{AB}_{2}$ in Fig. 1(a). Therefore, fluorination can most likely proceed from the opposite vertex or nearest sites $\left(\mathrm{AB}_{2}\right.$ and $\mathrm{AB}_{1}$ configuration). The stability increases with the concentration of fluorine adatoms, and the covalent nature tends towards the maximum attainable limit of $\mathrm{sp}^{2.43}$.

\subsection{Stability of the $F_{2}$ admolecule on graphene}

Previously, binding energies of $\mathrm{F}_{2}$ admolecules on graphene have been studied using two different geometrical optimization strategies: (i) In Ref. [60], it is assumed that the molecular bond length of the isolated molecule cannot be significantly changed during the adsorption process, since the intramolecular binding should be much stronger than the molecule surface interaction. Therefore, the equilibrium distance was found from a set of energy calculations for different heights of the $\mathrm{F}_{2}$ molecules (with fixed $\mathrm{F}-\mathrm{F}$ bond length) above the graphene surface. (ii) On the other hand, in Ref. [61] full relaxation has been carried out for an $\mathrm{F}_{2}$ molecule initially 
placed $1.8 \mathrm{~A}^{\circ}$ above the graphene surface, while interatomic $\mathrm{F}-\mathrm{F}$ distances were inferred according to $\mathrm{C}(\mathrm{F})-\mathrm{C}(\mathrm{F})$ separation for each configuration.

Since the geometry of the molecule near the surface may severely affect the binding energy of the compound, we have tried both initialization strategies, which favor one or the other of the geometrical analyses in Refs [60] and [61].

In the first approach, we relax an isolated $\mathrm{F}_{2}$ molecule. The PBE F-F bond length is found to be $1.42 \mathrm{~A}^{\circ}$, which agrees with experiment. We then bring it to a distance of $1.8 \mathrm{~A}^{\circ}$ from the graphene surface in the middle of a $\mathrm{C}-\mathrm{C}$ bond. According to Ref. [60], the parallel direction is more stable than the perpendicular one. In contrast to Ref. [60], we let the whole compound fully relax with rigorous convergence parameters. In the second approach, we use the strategy introduced in Ref. [61], but since the inter-fluorine atom distances in some of configurations are much larger than in isolated $\mathrm{F}_{2}$, we have placed the molecule initially at $3 \mathrm{~A}^{\circ}$ (the limit between physisorption and chemisorption) from the graphene surface, and again fully relaxed the combined structure. At small initial distances from graphene and large F-F separations, the molecule simply dissociates and adsorbs atomically on the surface, as happens for most compounds in Ref. [61], with the notable exception of the $\mathrm{AB}_{1}$ configuration.

From the two approaches, we obtain the same final geometry for $A A, A_{1}$ and $A_{2}$ configurations. From the first approach the interatomic distance in the fluorine molecule after full relaxation increases from $1.417 \mathrm{~A}^{\circ}$ to more than $1.8 \mathrm{~A}^{\circ}$. As a result, the binding energies shown in Table 4 are about three times larger than those obtained in Ref. [60]. In addition, contrary to Ref. [61], the fluorine molecules in $\mathrm{AA}, \mathrm{AB}_{1}$ and $\mathrm{AB}_{2}$ configurations do not dissociate, relaxing to a different stable distance from the graphene surface for each configuration: see Table 4. For the $\mathrm{AB}_{3}$ configuration the $\mathrm{C}(\mathrm{F})-\mathrm{C}(\mathrm{F})$ distance is much larger than the isolated $\mathrm{F}_{2}$ bond length $\left(3.75 \mathrm{~A}^{\circ}\right)$, so we applied the first approach. The interatomic distance of an $F_{2}$ isolated molecule increases from $1.417 \mathrm{~A}^{\circ}$ into $1.948 \mathrm{~A}^{\circ}$, while the $\mathrm{F}_{2}$ molecule is pushed up to a height of 2.544 $\mathrm{A}^{\circ}$, parallel to the initial graphene plane. 


\begin{tabular}{cccccccc}
\hline Configuration & $C(F)_{z}$ & $F_{z}$ & $C(F)-F$ & $F-F$ & Charge & $M$ & $\Delta E_{B}$ \\
& $A^{\circ}$ & $A^{\circ}$ & $A^{\circ}$ & $A^{\circ}$ & $F$ & $\left(\mu_{B}\right)$ & $(e V)$ \\
\hline $\mathrm{AA}$ & 0.031 & 2.638 & 2.620 & 1.952 & 0.387 & 1.25 & 0.7433 \\
$\mathrm{AB}_{1}$ & -0.002 & 2.705 & 2.713 & 1.816 & 0.357 & 0.75 & 0.7010 \\
$\mathrm{AB}_{2}$ & 0.012 & 2.624 & 2.651 & 1.950 & 0.371 & 1.25 & 0.7555 \\
$\mathrm{AB}_{3}$ & -0.096 & 2.544 & 2.716 & 1.948 & 0.412 & 1.25 & 0.7385 \\
\hline \hline
\end{tabular}

Table 4. Characterization of the ground state of an $\mathrm{F}_{2}$ admolecule above different positions on graphene (see Fig. 1). $\mathrm{C}(\mathrm{F})_{z}$ and $\mathrm{F}_{\mathrm{z}}$ are the z-coordinates of the atoms after relaxation (initially the graphene layer lies in the plane $z=0) . \mathrm{C}(\mathrm{F})-\mathrm{F}$ is the final bond length between the $\mathrm{C}(\mathrm{F})$ and fluorine atoms. $\mathrm{F}-\mathrm{F}$ is the final interatomic distance of the $\mathrm{F}_{2}$ molecule. The charge on each fluorine atom is given in units of electron charge $e . M$ is the total magnetic moment of the supercell in units of $\mu_{\mathrm{B}}$. The binding energy $\Delta \mathrm{E}_{\mathrm{B}}$ of the molecule on the surface is given in $\mathrm{eV}$. All distances are in $\mathrm{A}^{\circ}$.

According to our comparison of the two approaches, the equilibrium position of the $\mathrm{F}_{2}$ molecule is around $2.7 \mathrm{~A}^{\circ}$ above the graphene surface, as shown in Table 4 . The most stable configuration features $F_{2}$ in the $A_{2}$ arrangement. Here $A A$ is intermediate, and $A B_{1}$ shows the smallest $F-F$ distance (1.816 $\AA)$, leading to the weakest binding between the molecule and the surface ( 0.70 $\mathrm{eV})$. For an isolated $\mathrm{F}_{2}$ molecule and graphene we have a magnetic moment $M=0$. For $\mathrm{F}_{2}$ close to graphene $M$ increases, due to the weakened fluorine bond and hybridization. The smallest value of $M=0.75 \mu_{\mathrm{B}}$ appears for $\mathrm{AB}_{1}$, at the largest distance from the surface.

In agreement with Ref. [60], the $\mathrm{C}-\mathrm{F}$ bond length and the charges on each $\mathrm{F}$ atom show an ionic contribution to the binding energy, due to the charge transfer from graphene to the molecule: see Table 4. Although the two approaches give the same result, one should note that with increasing temperature $T$ the molecule may come closer to the surface. If the initial interatomic F-F distance is larger than in the isolated molecule, dissociation and atomic adsorption is possible, as our MD results confirm at $T=100 \mathrm{~K}$. Overall, we find the first approach to tracking molecular dissociation to be more reliable than the second one.

\subsection{Stability and diffusion of fluorine adatoms on graphene}

In the previous section the stabilities of FG were studied at lower coverages of fluorine. Now, we will see how the less stable configurations may transform to more stable ones through diffusion of $\mathrm{F}$ atoms. Previously, a barrier height of $290 \mathrm{meV}$ for migration of a single fluorine adatom to the nearest site has been found in calculations performed in a $4 \times 4$ supercell [54]. A barrier height of $356 \mathrm{meV}$ obtained in a $2 \times 2$ supercell using vdW-DF calculations has also been 
reported [23]. We obtain a smaller barrier height of $249 \mathrm{meV}$ to the diffusion of a single adatom to its nearest site on a $5 \times 5$ supercell. The residual difference with the previous results of [54], at the same theoretical level and functional, is probably the result of finite size effects.

There are different scenarios for the two-adatom case, depending on the position of the second adatom compared to the first one. Due to the computational cost of NEB calculations in a $5 \times 5$ supercell, we only consider diffusion to transform the $A A$ into the $\mathrm{AB}_{2}$ and $\mathrm{AB}_{1}$ configurations. Transforming from $\mathrm{AA}$ to either $\mathrm{AB}_{1}$ or $\mathrm{AB}_{2}$ happens through the first nearest neighbor: see Fig. 3. The corresponding barrier height in a $2 \times 2$ supercell is $47 \mathrm{meV}$ in [23]. The energy barriers per equal distance are shown in the same figure. NEB calculations show that diffusion completes only thanks to temporary local defects forming on the substrate along the path of the adatom. The transition of an $\mathrm{F}$ atom to its first nearest neighbor from an $\mathrm{A}$ to a $\mathrm{B}$ site takes place predominantly along a pathway forming an $\mathrm{AB}_{2}$ arrangement with an activation energy of $96 \mathrm{meV}$, which is 153 $\mathrm{meV}$ smaller than the diffusion barrier for a single adatom. The energy gain is $804 \mathrm{meV}$ and FG will quickly adopt the most stable configuration.

By using the Arrhenius law, the equivalent activation temperature is $T=\frac{\Delta E_{h}}{k_{B} \ln \frac{\Gamma_{0}}{\Gamma}}$. The factor $\Gamma_{0}$ is simply approximated by a typical phonon frequency of C-F stretching mode $\left(1000-1400 \mathrm{~cm}^{-}\right.$ ${ }^{1}$ ), i.e., $\sim 10 \mathrm{THz}$. That means $10^{13}$ attempts are performed per second by a fluorine adatom to jump from one site in sublattice $A$ into the nearest site from sublattice $B$. The equivalent estimated activation temperature of a successful jumping per second $\left(\Gamma=1 \mathrm{~s}^{-1}\right)$ is found to be about $40 \mathrm{~K}$ and the diffusion can readily happen at room temperature at a rate of $\sim 10^{11} \mathrm{~s}^{-1}$ or time scale of $\sim 10 \mathrm{ps}$. The reverse path from $\mathrm{AB}_{2}$ into an $\mathrm{AA}$ dimer requires a large activation energy, $900 \mathrm{meV}$, of the order of $\Delta \mathrm{E}_{\mathrm{B}}$.

We find that the energy barrier to diffuse an adatom to its first nearest neighbor (from AA to $\mathrm{AB}_{1}$ ) is about three times larger than from $\mathrm{AA}$ to $\mathrm{AB}_{2}$, with a time scale of $\sim 10$ ns: see Fig. 3 . Therefore, diffusion along a path using $\mathrm{AB}_{1}$ configurations is much less probable than through $\mathrm{AB}_{2}$. The same result has been reported for a small $2 \times 2$ supercell, using a vdW-DF functional [23]. The binding energies per adatom, given in Table 2, are much higher than the diffusion barrier. Therefore, the adatom cannot leave the surface during the diffusion. We note that a negative $\Delta U_{B}$ controls the barrier heights in diffusion. 


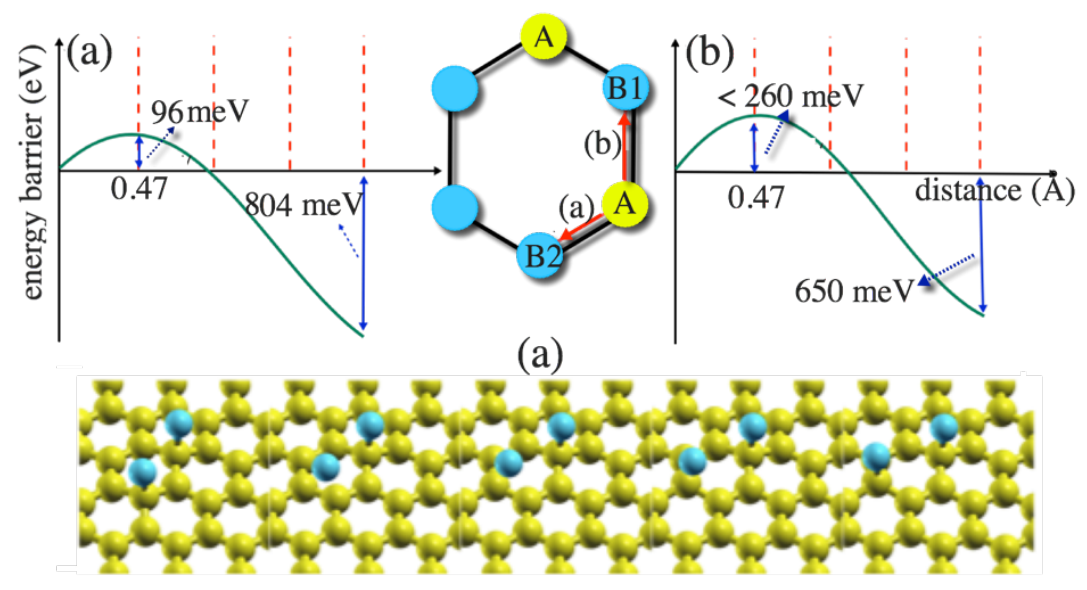

(b)

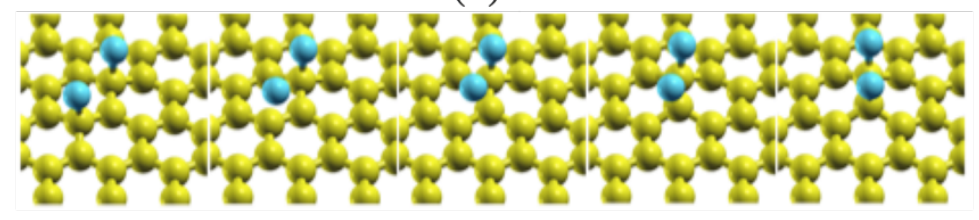

Fig. 3. (Color online) PBE-PAW predicted potential energy profiles and energy gains of the AA to (a) $A_{2}$ and (b) $A_{1}$ ad-dimer diffusion pathways. Selected NEB images along the corresponding pathways are shown in the bottom insets.

It is worth noting that hydrogen atoms experience an energy barrier of $0.50 \mathrm{eV}$ [35], twice as high as fluorine, to diffuse in a path from $A A$ into $\mathrm{AB}_{1}$. The more ionic nature of the $\mathrm{C}-\mathrm{F}$ bond is responsible for the distinct features of fluorine on graphene. Two unpaired electrons of AA configuration are delocalized through the B sublattice, and interact with the charge on fluorine atoms. During diffusion of fluorine from AA to each AB configuration, the electron spins will be paired, and the repulsive interaction reduced. On the other hand, larger charge depletion will occur around the $\mathrm{C}(\mathrm{F})$ atom, increasing the attractive interaction. These distinct features result in a smaller barrier height for diffusion of fluorine from the AA to the AB configuration on graphene compared to hydrogen.

We employ NEB calculations to study diffusion of fluorine atoms to transform an $\mathrm{AAA}_{1}$ configuration of three adatoms into the more stable $\mathrm{ABA}_{1}$ configuration. We find an energy barrier of $170 \mathrm{meV}$, smaller than for transforming $\mathrm{AA}$ to $\mathrm{AB}_{1}$, see Fig. 4, while the energy gain is $1.31 \mathrm{eV}$. The corresponding activation temperature is $61 \mathrm{~K}$ and diffusion happens on a time scale of $\sim 100$ ps at room temperature. 


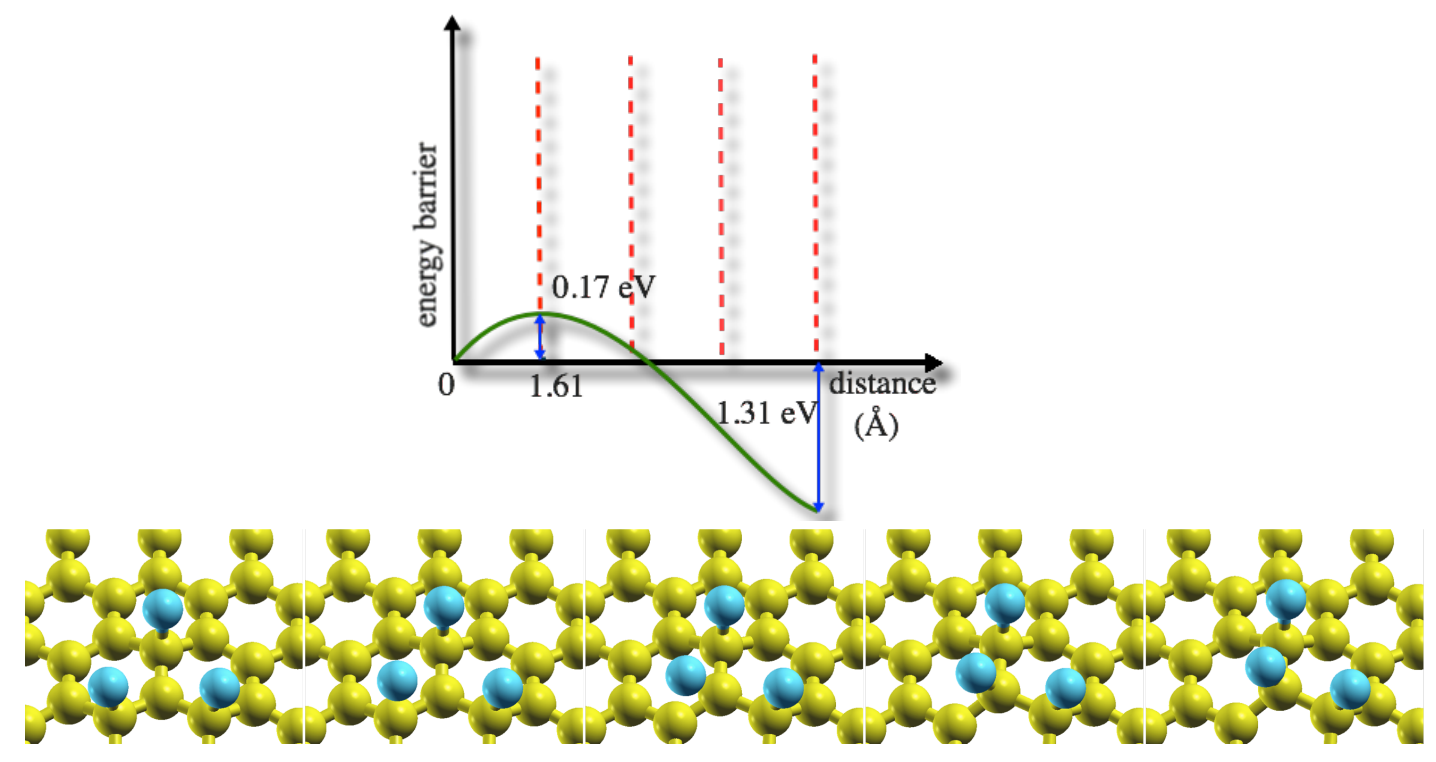

Fig. 4. (Color online) PBE-PAW predicted potential energy profile and energy gain of the $\mathrm{AAA}_{1}$ to $\mathrm{ABA}_{1}$ ad-trimer diffusion pathway. Selected NEB images along the pathway are shown in the bottom insets.

The diffusion process to reach the most stable configuration follows the $\Delta U_{B}$ parameter. As seen in Table 2, $\Delta \mathrm{U}_{\mathrm{B}}$ decreases considerably from AA into AAA configurations. $\Delta \mathrm{U}_{\mathrm{B}}$ is influenced by the ionic character of the $\mathrm{C}-\mathrm{F}$ bond and therefore, we expect to be able to decrease the diffusion barrier height by adding adatoms on the same sublattice.

\subsection{Stability and total magnetic moment}

Fluorine has an unpaired electron, which carries a spin moment of $1 \mu_{\mathrm{B}}$, and one expects that the breaking of a $\pi$ bond from the graphene network by a fluorine adatom will induce $1 \mu_{\mathrm{B}}$ magnetization on the structure. Experimental evidence shows that fluorine adatoms do indeed carry spin $1 / 2$ magnetic moments. However, no magnetic ordering could be identified down to liquid helium temperature, and the maximum measured magnetic response is limited to one moment per approximately 1,000 carbon atoms [40].

While theoretical studies of the magnetic properties of hydrogenated graphene show compatible results, the magnetic properties of FG in lower fluorine concentrations are a subject of debate and the issue is not fully understood. Based on GGA-DFT outcomes, due to the absence of the exchange splitting of impurity states, single $\mathrm{F}$ adatoms adsorbed on $\mathrm{n} \times \mathrm{n}$ supercells of graphene 
with $\mathrm{n} \geq 3$ (concentration equal or less than $\mathrm{CF}_{0.056}$ ) are predicted to be non-magnetic [24, 36-38]. In Ref. [24], this result has been attributed to the large F-F distance and short-range magnetic coupling, which may prevent adatoms from coupling even through the bulk $\pi$ states of graphene. On the other hand, in Ref. [36] the DFT-predicted $M=0$ state was justified by a model that includes the interactions for only F, $\mathrm{C}(\mathrm{F})$, and $\mathrm{C}(\mathrm{NN})$ atoms leading to an effective Anderson impurity, in which the absence of magnetism can be explained in terms of its effective parameters.

The above assertions are based on DFT predictions, while it has been shown previously that owing to self-interaction error (SIE), DFT local and semilocal exchange-correlation (xc) potentials cannot predict the correct spin state of an $\mathrm{H}$ adatom adsorbed on a flat graphene surface [62]. SIE is associated with the improper long-range asymptotic behavior of $\mathrm{xc}$ functionals and it plays a decisive role in charge-transfer systems and heteronuclear diatomic molecules (XY), when the lowest unoccupied molecular orbital (LUMO) energy of atom $Y$ lies below the highest molecular orbital (HOMO) of atom X [63-64]. C-H bonding, when one constrains the surface to be flat, is a reminiscent of ionic bonding. In such a situation, the energies of the occupied and unoccupied hydrogen $s$ orbitals have been shown to converge when approaching the graphene surface. Below a certain distance they become degenerate and approach the Fermi energy [62]. At this point, these orbitals are equally occupied by a fractional number of electrons, leading to spin quenching [62]. In contrast to hydrogen, the LUMO of a fluorine atom lies below the Fermi level of graphene. For semi-ionic C-F bonds, the DFT prediction of partial occupation of spin up and down near the Fermi energy, yielding $\mathrm{M}=0$, is dubious. Hybrid functionals can cure the SIE, but at great computational cost. $\mathrm{M} \approx 1 \mu_{\mathrm{B}}$ has been reported from HSE hybrid functional for a single fluorine adsorbed on a graphene supercell of $3 \sqrt{3} \times 3 \sqrt{3} R 30^{\circ}, \mathrm{CF}_{0.019}$ [39]. PBE $+\mathrm{U}$ is a less expensive remedy (described in detail in Ref. [65]) to predict correctly the magnetic response of the FG structure. We employ $\mathrm{PBE}+\mathrm{U}$ and investigate the relationship between the stability and magnetic response of the configurations shown in Fig. 1(a).

Total magnetizations are computed by integrating the components of the spin density, which are summed over all bands and $\mathbf{k}$-points. The predicted $\mathrm{M}$ values for configurations that have one or more broken $\pi$ bonds with imbalance between the number of sites of the two sublattices are not the same within the PBE and PBE+U methods, as can be seen in Table 2. PBE+U predicted partial density of states (pDOS) of $\mathrm{CF}_{0.02}$ show that the $\mathrm{p}_{z}$ orbitals of fluorine and carbon atoms 
determine the magnetic response of FG. The pDOS of $p_{x}$ and $p_{z}$ orbitals are shown in Fig. 5. No spin polarization is observed for $\mathrm{p}_{x}$ orbitals. Instead, a clear splitting of $\mathrm{p}_{z}$ spin up and down orbitals are observed, which induces magnetism in the structure via exchange interaction between itinerant $\mathrm{p}_{z}$ electrons, and makes them semilocalized. The exchange splitting $\Delta E_{X}$ is about 0.43 $\mathrm{eV}$, larger than $k_{\mathrm{B}} T$ at $T=300 \mathrm{~K}$, and satisfies the Stoner criterion with ferromagnetic ordering for the same-sublattice sites (ferrimagnetic in general). Therefore, one may expect a Curie $T_{C}$ above room temperature at this concentration, according to the Stoner criterion.
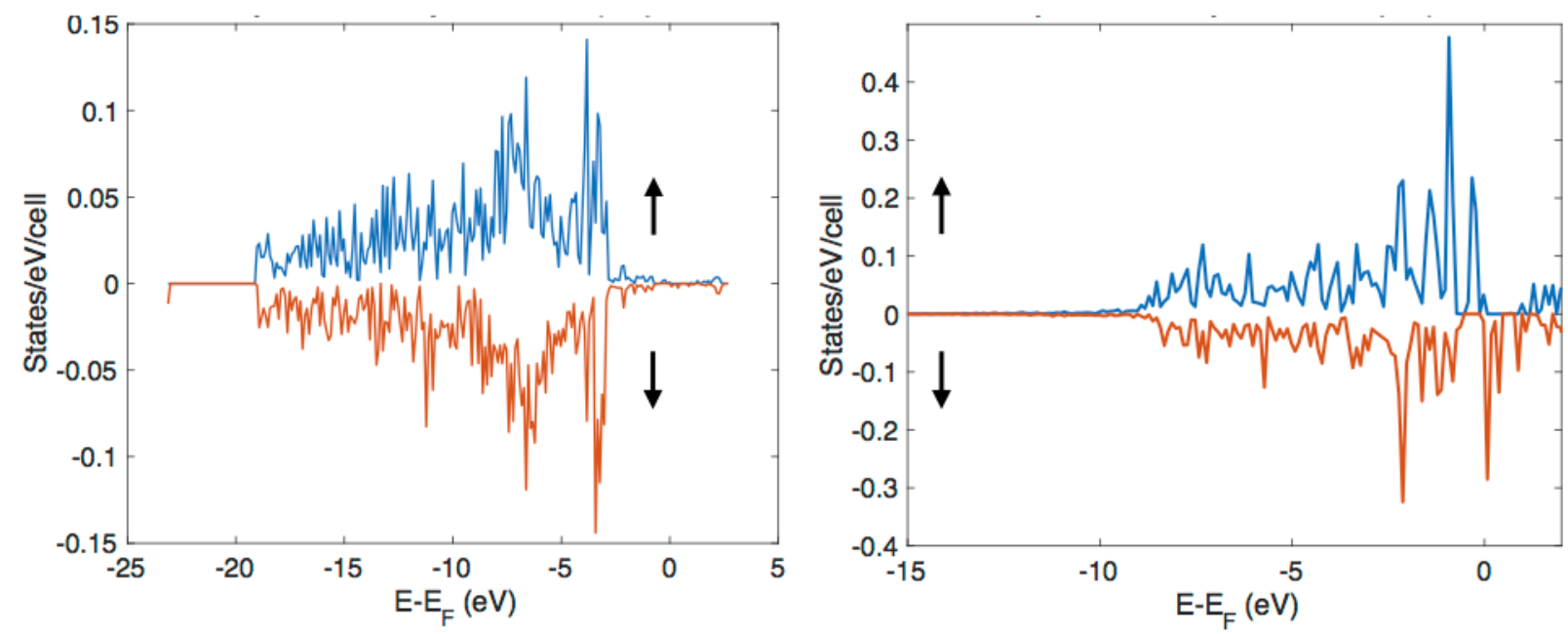

Fig. 5. (Color online) PBE+U predicted partial density of states (pDOS) of the majority (blue color) and minority (red color) electrons of $\mathrm{p}_{\mathrm{x}}$ (left panel) and $\mathrm{pz}$ orbitals (right panel). Almost no polarization for px orbitals is observed. Comparing pDOS of occupied pz and px orbitals of majority and minority electrons shows the magnetic properties of FG mostly stem from pz orbitals for structures with an imbalance in number of adatoms between two sublattices. Fermi energies $\mathrm{E}_{\mathrm{F}}$ are set to zero.

In Fig. 6(a), we report an isosurface of the spin density distribution predicted by $\mathrm{PBE}+\mathrm{U}$ for $\mathrm{CF}_{0.02}$. Assuming the fluorine is adsorbed on a carbon belonging to the A sublattice, we observe that most of the $1 \mu_{\mathrm{B}}$ of magnetization, previously carried by a fluorine atom, is now distributed symmetrically with long-range polarization on carbon atoms of opposite sublattice $B$. The highest spin density comes from $\mathrm{C}(\mathrm{NN})$ atoms and gradually decays with the distance from adsorption site. Small negative magnetization is observed on carbons from the A sublattice, which has spins antiparallel to the fluorine atom. The resulting total magnetization predicted by $\mathrm{PBE}+\mathrm{U}$ is $\mathrm{M}=1 \mu_{\mathrm{B}}$. For adsorption of a fluorine dimer on a $5 \times 5$ supercell of graphene, $\mathrm{CF}_{0.04}$, two different 
ground states with singlet $(\uparrow \downarrow)$ and triplet $(\uparrow \uparrow)$ spin arrangements are possible. In FG with the $\mathrm{AB}_{1}$ configuration, the $\mathrm{C}(\mathrm{NN})-\mathrm{C}(\mathrm{F})$ bond length of the singlet state is closer to the carbon $\mathrm{sp}^{3}$ characteristic bond length (1.49 $\AA$ against $1.47 \AA$ ). In addition, $\Delta U_{B}$ takes a negative value for an "unpaired" triplet spin arrangement. In the triplet case, the two parallel spins further avoid each other through exchange interactions and the stability of the triplet state is reduced by another 1.5 $\mathrm{eV}$. Accordingly, clustering can only be made from singlet state and both DFT and DFT $+\mathrm{U}$ predicts $A_{1}$ and $A_{2}$ are non-magnetic structures. Here no antiferromagnetic coupling is even observed between adjacent sites, instead $\mathrm{M}=0 \mu_{\mathrm{B}}$ is a result of nearly zero magnetic dipole on each site which resembles the magnetic feature of pristine graphene.

(a)

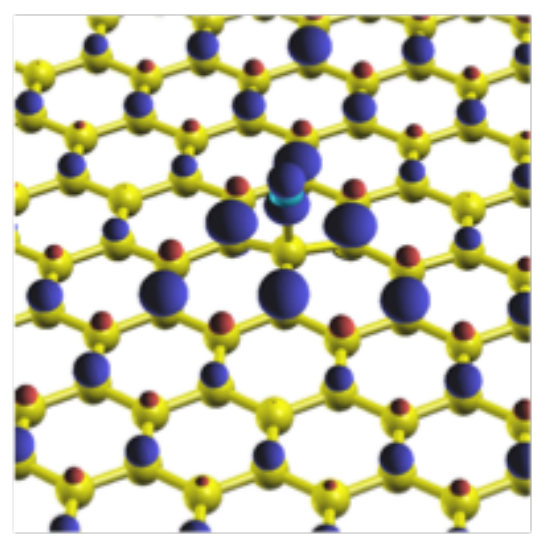

(b)

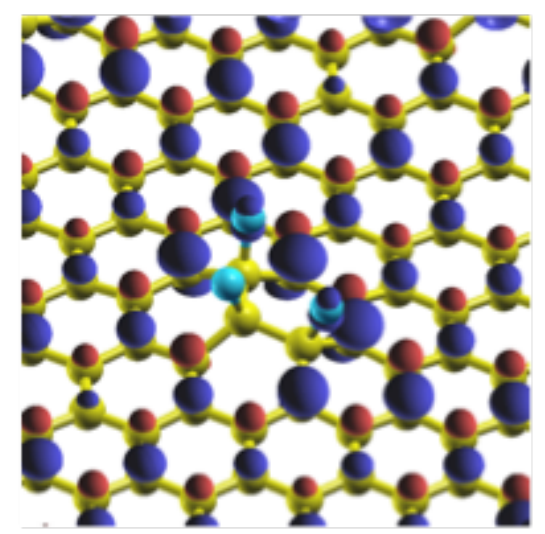

Fig. 6. (Color online) (a) Spin density isosurface of a single F adatom on graphene. Carbon atoms are shown in yellow and fluorine atoms in blue. If the fluorine atom binds to a carbon atom $\mathrm{C}(\mathrm{F})$ on an A site, the spin-up excess (dark blue bubbles) is observed on the B sublattice and the spindown excess (red bubbles) is found on the A sublattice. The largest contribution is from the nearest neighbors $\mathrm{C}(\mathrm{NN})$ to the $\mathrm{C}(\mathrm{F})$ atom. (b) Spin density isosurface of an $\mathrm{ABA}_{1}$ ad-trimer on graphene. Two $\mathrm{F}$ adatoms are coupled ferromagnetically with each other and antiferomagneticaly with the third adatom bonded to the B sublattice.

In an AA configuration two adatoms are unevenly distributed between the two sublattices ( $\mid N_{A}$ $N_{B} \mid=2$, where $N_{\mathrm{A}}$ and $N_{\mathrm{B}}$ are the numbers of defects on sublattices A and B, respectively). According to Lieb's theorem [66] (with a pictorial explanation in Fig. 7), total spin of the FG is equal to half the imbalanced in the numbers of adatoms on each sublattice: $S=\left|N_{A}-N_{B}\right|=2$. Therefore, in contrast to the AB configuration, ferromagnetic ordering with $S=1$ is the only 
possible choice for the magnetism originating from two fluorines bonding to the same sublattice. $M=2 \mu_{\mathrm{B}}$ has been shown for AA hydrogen configuration [33-34]. PBE-GGA predicts a small non-zero $M$ for the AA FG configuration, while $\mathrm{PBE}+\mathrm{U}$ predicts a total magnetization of less than $M=1.6 \mu_{\mathrm{B}}$ (smaller than the expected $M=2 \mu_{\mathrm{B}}$ ). It is probable that the semi-ionic nature of the $\mathrm{C}-\mathrm{F}$ bond makes electrons on the impurity states less localized and gives rise to smaller $M$, compared to the AA hydrogen configuration. This point should be examined in more detail in the future, with SIE corrected functionals. In any event, AA with about $M \approx 2 \mu_{\mathrm{B}}$ is not a favorable configuration according to the $\Delta U_{B}$ stability criterion. Therefore, non-magnetic structure is the most likely configuration during the fluorination process.

(a)

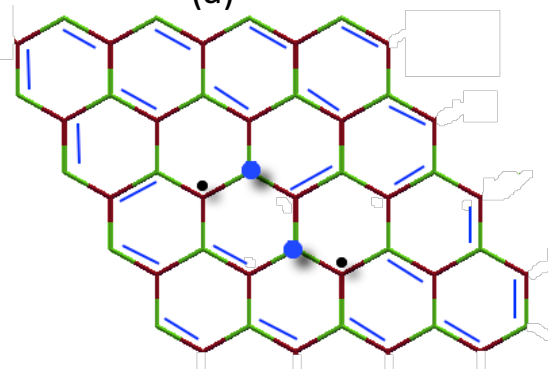

(b)

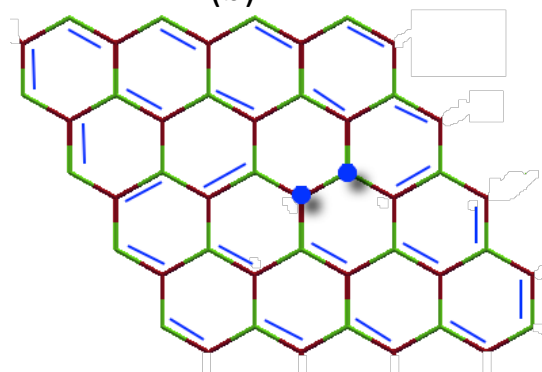

Fig. 7. (Color online) Resonating valence bond model of the itinerant electron in a $5 \times 5$ unit cell of graphene for $\mathrm{AA}$ and $\mathrm{AB}_{1}$ ad-dimer. Adatoms are shown in blue circles and nonbonded electrons with black dots. The two sublattices $A$ and $B$ are distinguished from each other by the green color for sublattice A and red for sublattice B. There are two and one broken $\pi$ bonds, respectively, in panels (a) and (b).

We select three different configurations $\left(\mathrm{ABA}_{1}, \mathrm{AAA}_{1}\right.$ and $\left.\mathrm{AAA}_{2}\right)$ to inspect the magnetic response of three fluorine adatoms adsorbed on a $5 \times 5$ cell of graphene, modeling $\mathrm{CF}_{0.06}$, with constrained atomic magnetizations. PBE-GGA calculations for the $\mathrm{ABA}_{1}$ configuration predicts that the $\uparrow \downarrow \uparrow$ spin arrangement is preferred over $\uparrow \uparrow \uparrow$ by about $0.75 \mathrm{eV}$ per adatom and gives a total magnetization of $M=0.48 \mu_{\mathrm{B}}$. $\mathrm{PBE}+\mathrm{U}$ predicts that two adatoms in the A sublattice are coupled ferromagnetically with each other, and antiferromagnetically with the third adatom in B sublattice, see Fig. 6(b), resulting in $M=1 \mu_{\mathrm{B}}$. Spin-up excess is distributed with the same pattern around two adatoms bounded to carbon atoms on the B sublattice, whereas spin-down excess is distributed on the A sublattice. While PBE-GGA predicts $M=1 \mu_{\mathrm{B}}$ for the $\mathrm{AAA}_{1}$ and $\mathrm{AAA}_{2}$ configurations, $\mathrm{PBE}+\mathrm{U}$ predicts that three adatoms are coupled ferromagnetically, resulting in 
$M=3 \mu_{\mathrm{B}}$.

Consequently, the magnetic response of graphene is influenced by its bipartite nature and follows the symmetry of the defect sites. For each adatom on one sublattice, an electron of the opposite sublattice will be unpaired, which breaks the equivalence between the two sublattices. According to the bipartite symmetry only electrons of two opposite sublattices can be paired. By using a pictorial sketch of resonant valence bond model (Kekulé) structures, shown in Fig. 7, one can explain simply the bipartite magnetic response of graphene. As an example, when two fluorine atoms are bound to two carbon atoms on the same sublattice $A$, two $p_{z}$ electrons with the same spin alignments are left on the B sublattice. Instead, there is no unpaired electron in Fig. 7(b). When two adatoms are bonded to opposite sublattices, only one $\pi$ bond is broken in the graphene network.

Therefore, evenly mixed sublattice binding configurations have higher stability and $M \sim 0$ with non-magnetic feature. Our model explains why the maximum detected magnetic response has been limited to approximately one moment per 1,000 carbon atoms [40]: fluorination will develop geometrically from a central carbon, and additional fluorine adatoms will bind to the nearest neighbor atoms (and so on) to avoid the imbalance between two sublattices, leading to the minimum possible total magnetization.

\subsection{Electronic properties of adatoms on graphene}

The electronic behavior of FG is severely influenced by the type (single- or double-side fluorination), density and arrangement of fluorine adatoms on the surface. Our study of singlesided FG shows different electronic behavior from published results on double-sided FG. The experimental reported double-sided fully FG band gap is $3 \mathrm{eV}$ [15] and is in agreement with the theoretical prediction of $2.96 \mathrm{eV}[16,67]$. 

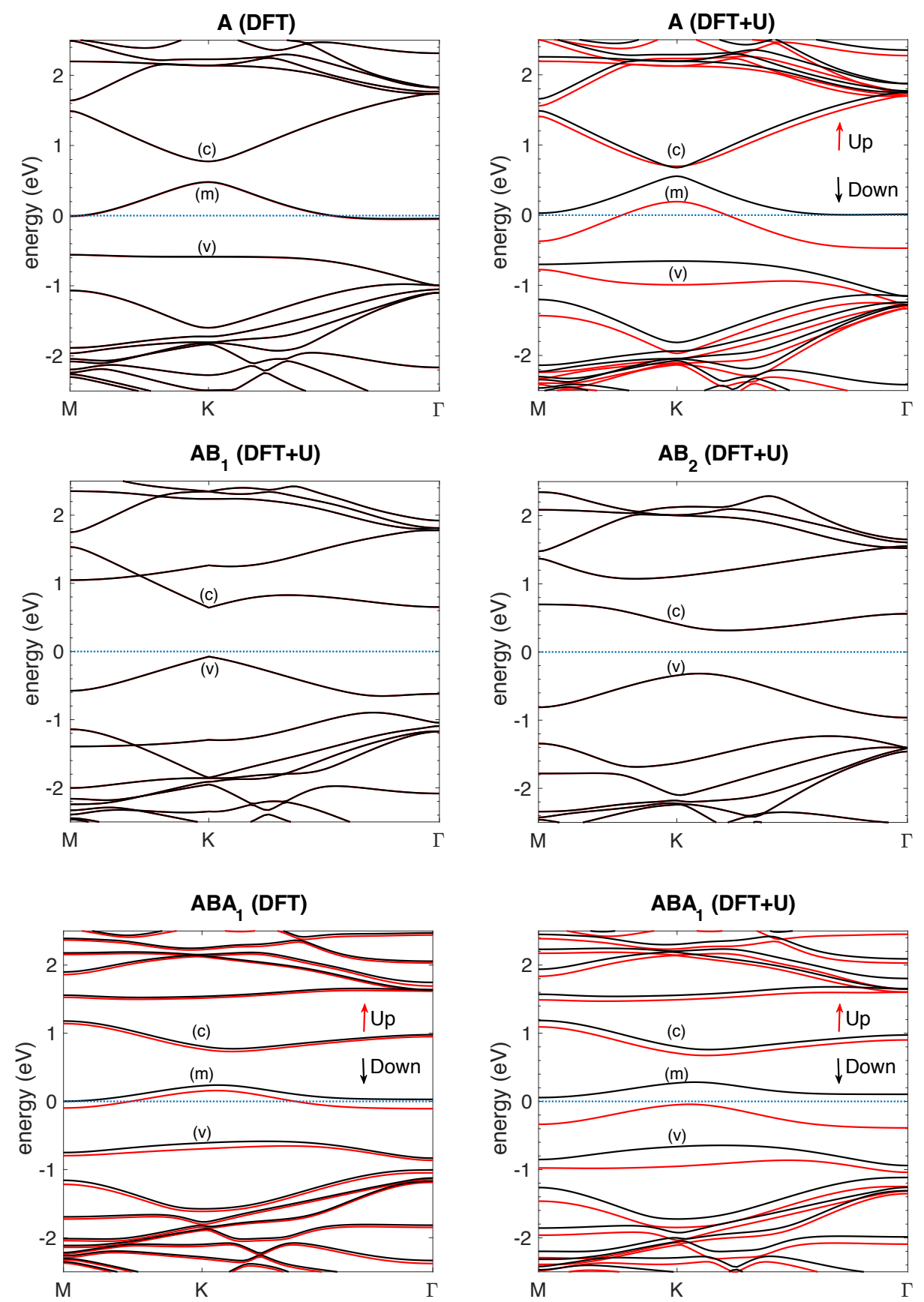

Fig. 8. DFT and DFT $+\mathrm{U}$ band structures of different configurations of single-sided FG. The energies are plotted relative to the Fermi level (blue dotted line). For $\mathrm{AB}_{1}$ and $\mathrm{AB}_{2} \mathrm{DFT}$ and $\mathrm{DFT}+\mathrm{U}$ give the same result.

Single-sided $\mathrm{C}_{2} \mathrm{~F}$ chair was predicted as a gapless metal, but the $\mathrm{C}_{2} \mathrm{~F}$ boat arrangement is a semiconductor with a band gap of $1.57 \mathrm{eV}$. A theoretical band gap of $2.93 \mathrm{eV}$ is predicted for 
single-sided $\mathrm{CF}_{0.25}$ in $\mathrm{C}_{4} \mathrm{~F}$ arrangement [16], and experimental evidence confirm that single-sided fluorine adatom, saturated at $\% 25$, is optically transparent (which implies a band gap of about 3 $\mathrm{eV}$ ) and is over six order of magnitude more resistive than graphene [19]. Since DFT predicts a band gap of $2.93 \mathrm{eV}$ for $\mathrm{C}_{4} \mathrm{~F}$, the optical transparency found experimentally at this concentration of adatoms was interpreted as the probable arrangement of fluorine adatoms in $\mathrm{C}_{4} \mathrm{~F}$ configuration [19]. On the other hand, electrical properties of large area few-layer graphene, subjected to plasma-induced fluorination from one side, show strong localization, which emerges as fluorination progresses. In addition, the initial metallic behavior of graphene changes to insulating, with the highest band gap of $\sim 80 \mathrm{meV}$ for the most fluorinated structure [18]. The small band gap of single-sided FG in Ref. [18] does not disagree with the optical transparency of single-sided fluorinated graphene saturated at $\% 25$ concentration, since these are probably two different concentrations and configurations.

Here we present the electronic behavior of the most stable structures $A, A_{1}, A B_{2}, A B A_{1}$. The detailed role of $\mathrm{C}-\mathrm{F}$ bonds and graphene sublattices on the electronic features will be presented in another paper. The effect of fluorine adatoms on the electronic properties of graphene depends on the concentration. At low concentration, FG is a p-doped half-metal; at high concentration it is a gapped semiconductor.

Also, the electronic behavior is naturally and strongly linked to the magnetic properties. Configurations with zero magnetic moment $\left(\mathrm{AB}_{1}\right.$ and $\mathrm{AB}_{2}$ with $\left.\mathrm{CF}_{0.04}\right)$ are semiconductors within both DFT and DFT $+\mathrm{U}$. The band structure and band gap are influenced not only by the fluorine concentration, but also by the adatom arrangement in the cell. The electrophilic character of fluorine causes Fermi level shifts into the valence Dirac cone by about $0.70 \mathrm{eV}$. This results in a p-type semiconductor for $\mathrm{AB}_{1}$. A linear dispersion around $\mathrm{K}$ is still observed for $\mathrm{AB}_{1}$, corresponding to extended $\pi$-bonding of $2 \mathrm{p}_{\mathrm{z}}$ orbitals. $\mathrm{AB}_{2}$ is an intrinsic semiconductor with a band gap of about $0.63 \mathrm{eV}$. In contrast to $\mathrm{AB}_{1}$, the linear dispersion around $\mathrm{K}$ becomes parabolic due to symmetry breaking and avoided crossings, which correspond to stronger orbital hybridization of $\mathrm{F}-\mathrm{C}$ bonds and larger local defect radii of $\mathrm{AB}_{2}$, compared to $\mathrm{AB}_{1}$.

In addition to a magnetic moment, a sublattice imbalance creates midgap states around the Fermi level: the number of midgap states is equal to the number of uncompensated sites between 
the two A and B sublattices $\left(\left|N_{\mathrm{A}}-N_{\mathrm{B}}\right|\right)$. The DFT predicted band structure of $\mathrm{CF}_{0.02}$, A structure, with one midgap state shown in Fig. 8 does not display significant splitting between spin-up and down states. According to DFT, fluorine splits the valence and conduction bands by $1.33 \mathrm{eV}$ at the K-point, while the overall structure is metallic. The gap between the conduction and midgap states at the K-point is only $0.30 \mathrm{eV}$. According to DFT+U, fluorine shifts down the energy of the majority-spin electrons and hence splits the spin-up and down levels by $1.33 \mathrm{eV}$, as expected on physical grounds, while the gap between valence and conduction bands of FG at the K-point is $1.69 \mathrm{eV}$. Both DFT and DFT+U confirm that fluorine donates holes to graphene in $\mathrm{CF}_{0.02}$, so that the Fermi level is pushed down, but from DFT $+U$ the structure is a half-metal in the spin up channel as seen in Fig. 8. Within DFT+U, all k-states of spin down midgap states are at least 5 meV above the Fermi level at $T=0$. The overall small indirect gap of $5 \mathrm{meV}$ between the highest occupied and the lowest unoccupied states occurs between spin-up and spin-down midgap states.

Now we consider a higher fluorine concentration. The shape of the band structure of $\mathrm{ABA}_{1}$ $\left(\mathrm{CF}_{0.06}\right)$ (one uncompensated site) is similar to that of the A structure, and the symmetry of the two graphene sublattices plays a dominant role in the electronic properties. In the $\mathrm{ABA}_{1}$ case, however, the band at $\mathrm{K}$ is almost flat and the Dirac-cone structure is completely disturbed. The bandwidth of the midgap state is decreased from $0.55 \mathrm{eV}$ in $\mathrm{A}$ to $0.35 \mathrm{eV}$ in $\mathrm{ABA}_{1}$. This is a sign of stronger localization of electrons, and even a pure DFT functional, which often suffers from an electron delocalization error, can retrieve here a spin-splitting energy of about $0.13 \mathrm{eV}$ and predicts $M=0.47 \mu_{\mathrm{B}}$ (see Table 2). The corresponding spin splitting of the midgap state is $0.49 \mathrm{eV}$ in DFT+U. As in the A structure, the majority spin channel (up) is shifted down in energy (Fig. 8 by comparing DFT and DFT $+\mathrm{U})$. DFT predicts the structure to be a half-metal at $T=0$, and energies of all points corresponding to minority channel in midgap states are located at least 2 meV above the Fermi level in the ground state. On the other hand, DFT $+U$ predicts $\mathrm{ABA}_{1}$ to be a p-type semiconductor with an indirect band gap of about $98 \mathrm{meV}$. The energies of all points corresponding to majority channel of midgap states are located at least $43 \mathrm{meV}$ below the Fermi level. Comparing the band structure of $\mathrm{A}$ and $\mathrm{ABA}_{1}$ shows that the band gap in single-sided FG with uncompensated sublattices increases slowly with fluorine density, and the band gap we obtain is of the same order of magnitude as experiment $(80 \mathrm{meV})$ [18].

\section{Conclusion}


We have studied the stability, dynamics, magnetic behavior and electronic structure of $\mathrm{F}$ adatoms on graphene. We show that the finite-size error in the binding energy of isolated fluorine adatoms in a periodic supercell stems from the interaction of the electric dipole moments of the images of the defects, (as expected if the response of graphene were that of a perfect metal), and falls off as the inverse cube of the linear cell size. Therefore, we can obtain the finite-sizecorrected binding energy of a single fluorine adatom in the infinite dilute limit.

In contrast to hydrogenated graphene, the formation energy of single-sided FG is favorable, which implies FG is more stable than hydrogenated graphene at higher temperatures. The $\mathrm{C}-\mathrm{F}$ bond nature was found to be semi-ionic in nature for low concentrations of fluorine. The covalent nature increases with the fluorine concentration, from $\mathrm{sp}^{2.33}$, with a maximum attainable limit of $\mathrm{sp}^{2.43}$ in covalent nature. We find that the standard binding and formation energies are not sufficient criteria to study the stability of clusters of fluorine adatoms on graphene. The stability of the structures is further characterized by the binding energy with respect to dissociation into isolated adatoms $\left(\Delta U_{B}\right)$. For multiple fluorine adatoms, those configurations in which adatoms bond evenly to both sublattices are the most stable, and the stability increases with concentration. We have checked that $\mathrm{F}_{2}$ admolecules are also stable (though less than atomic $\mathrm{F}$ ) and have slightly ionic bonding.

The semi-ionic character of $\mathrm{C}-\mathrm{F}$ bonds imposes specific features in FG, such as smaller diffusion barriers, compared to hydrogenated graphene. We show that $\mathrm{F}$ atoms are mobile on a graphene surface, through pathways that lead fluorine atoms to the most stable configurations. The diffusion probability is influenced by the binding energy and $\Delta U_{B}$, and the barrier height decreases with increasing fluorination on sites of the same sublattice. Low activation energies, to transform structures into configurations with balanced sublattices, suggest fluorination proceeds with atoms successively binding to neighboring carbon atoms from opposite sublattices. We discuss how the bipartite feature of graphene prevents the formation of significant magnetic ordering during the fluorination process. Pairs of fluorine adatoms adopt configurations which do not perturb the balance of spins on the graphene sublattices, so that the magnetic behavior resembles that of pristine graphene. If the $\mathrm{F}$ sublattices are unpaired, the coupled $\mathrm{F}$ atom spin moments will arrange in order to minimize the total magnetization. Semilocal DFT fails to 
describe correctly the magnetic moment and electronic structure of FG, and a higher level theory is needed, such as DFT $+\mathrm{U}$. According to $\mathrm{DFT}+\mathrm{U}$, the electronic properties are dominated by splitting of the majority and minority channels, and the structures show p-type half-metallic or semiconducting features, depending on the adatom arrangements at a given concentration.

\section{Acknowledgements}

The authors gratefully acknowledge the computational assistance provided by Department of Energy Engineering and Physics at Amirkabir University of Technology. MJV acknowledges an A.R.C. grant (AIMED 15/19-09) from the Communaute francaise de Belgique and the Belgian

Fonds National de la Recherche Scientifique FNRS grant numbers PDR T.1077.15-1/7. F. Marsusi acknowledges Yalda Pedram and Sarina Yousofbeigi for setting up MD calculations.

\section{References}

[1] Han W, Kawakami RK, Gmitra M, Fabian J. Graphene spintronics. Nat nanotechnology 2014;9(10):794-807

[2] Neto AC, Guinea F, Peres NM, Novoselov KS, Geim AK. The electronic properties of graphene. Rev Mod Phys 2009;81(1):109.

[3] Rao CN, Voggu R. Charge-transfer with graphene and nanotubes. Materials today 2010;13(9):34-40.

[4] Das B, Voggu R, Rout CS, Rao CN. Changes in the electronic structure and properties of graphene induced by molecular charge-transfer. Chemical Communications 2008;(41):5155-57. [5] Limbu TB, Mendoza F, Barrionuevo D, Carpena J, Maruyama B, Katiyar RS, Weiner BR, Morell G. Study on the optical and electrical properties of tetracyanoethylene doped bilayer graphene stack for transparent conducting electrodes. AIP Advances 2016;6(3):035319.

[6] Varghese N, Ghosh A, Voggu R, Ghosh S, Rao CN. Selectivity in the interaction of electron donor and acceptor molecules with graphene and single-walled carbon nanotubes. J Phys Chem C 2009;113(39):16855-9. 
[7] Hou Y, Geng X, Li Y, Dong B, Liu L, Sun M. Electrical and Raman properties of p-type and n-type modified graphene by inorganic quantum dot and organic molecule modification. Science China Physics, Mechanics and Astronomy 2011;54(3):416-9.

[8] Panchakarla LS, Subrahmanyam KS, Saha SK, Govindaraj A, Krishnamurthy HR, Waghmare UV, Rao CNR. Synthesis, structure, and properties of boron-, nitrogen-doped graphene. Advanced Materials 2009;21(46):4726-30.

[9] Wang H, Maiyalagan T, Wang X. Review on recent progress in nitrogen-doped graphene: synthesis, characterization, and its potential applications. Acs Catalysis 2012; 2(5):781-4.

[10] Wu Z, Han Y, Huang R, Chen X, Guo Y, He Y, Li W, Cai Y, Wang N. Semimetallic-tometallic transition and mobility enhancement enabled by reversible iodine doping of graphene. Nanoscale 2014;6(21):13196-202.

[11] Hoyt RA, Remillard EM, Cubuk ED, Vecitis CD, Kaxiras E. Polyiodide-doped graphene. J Phys Chem C 2017;121(1):609-15.

[12] Kalita G, Wakita K, Takahashi M, Umeno M. Iodine doping in solid precursor-based CVD growth graphene film. J. Mater Chem 2011;21(39):15209-13.

[13] Tran NTT, Nguyen DK, Glukhova OE, Lin MF. Coverage-dependent essential properties of halogenated graphene: A DFT study. Scientific reports 2017;7(1),17858.

[14] Karlický F, Kumara Ramanatha Datta K, Otyepka M, Zbořil R. Halogenated graphenes: rapidly growing family of graphene derivatives. ACS nano 2013;7(8):6434-64.

[15] Nair RR, Ren W, Jalil R, Riaz I, Kravets VG, Britnell L, Blake P, Schedin F, Mayorov AS, Yuan S, Katsnelson MI. Fluorographene: a two-dimensional counterpart of Teflon. small 2010;6(24):2877-84.

[16] Şahin H, Topsakal M, Ciraci S. Structures of fluorinated graphene and their signatures. Phys Rev B 2011;83(11):115432.

[17] Nakada K, Ishii A. DFT calculation for adatom adsorption on graphene. In Graphene Simulation 2011. InTech.

[18] Zhao R, Jayasingha R, Sherehiy A, Dharmasena R, Akhtar M, Jasinski JB, Wu SY, Henner V, Sumanasekera GU. In Situ Transport Measurements and Band Gap Formation of Fluorinated Graphene. J Phys Chem C 2015;119(34):20150-5. 
[19] Robinson, JT, Burgess, JS, Junkermeier, CE, Badescu, SC, Reinecke TL, Perkins, FK, Zalalutdniov MK, Baldwin JW, Culbertson JC, Sheehan PE, Snow ES. Properties of fluorinated graphene films. Nano letters 2010;10(8):3001-5.

[20] Withers F, Russo S, Dubois M, Craciun M F. Tuning the electronic transport properties of graphene through functionalisation with fluorine. Nanoscale Res Lett 2011; 6(1):526.

[21] Lee WH, Suk JW, Chou H, Lee J, Hao Y, Wu Y, Piner R, Akinwande D, Kim KS, Ruoff RS. Selective-area fluorination of graphene with fluoropolymer and laser irradiation. Lett 2012;12(5):2374-8.

[22] Leenaerts O, Peelaers H, Hernández-Nieves AD, Partoens B, Peeters FM. First-principles investigation of graphene fluoride and graphane. Phys Rev B 2010;82(19):195436.

[23] Santos H, Henrard L. Fluorine adsorption on single and bilayer graphene: role of sublattice and layer decoupling. J Phys Chem C 2014;118(46):27074-80.

[24] Liu HY, Hou ZF, Hu CH, Yang Y, Zhu ZZ. Electronic and magnetic properties of fluorinated graphene with different coverage of fluorine. J Phys Chem C 2012;116(34):18193-201.

[25] Zhou S, Sherpa SD, Hess DW, Bongiorno A. Chemical bonding of partially fluorinated graphene. J Phys Chem C 2014;118(45):26402-8.

[26] Nguyen KD, Tran TN, Nguyen TT, Chiu YH, Lin MF. Essential Properties of Fluorinated Graphene and Graphene Nanoribbons. arXiv preprint arXiv:1712.03824. 2017 Dec 8.

[27] Karlický F, Zbořil R, Otyepka M. Band gaps and structural properties of graphene halides and their derivates: A hybrid functional study with localized orbital basis sets. J Chem Phys 2012;137(3):034709.

[28] Shi H, Pan H, Zhang YW, Yakobson BI. Electronic and magnetic properties of graphene/fluorographene superlattices. J Phys Chem C 2012;116(34):18278-83.

[29] Haddon RC. GVB and POAV analysis of rehybridization and $\pi$-orbital misalignment in nonplanar conjugated systems. Chem Phys Lett 1986;125(3):231-4.

[30] Haddon RC, Scott LT. $\pi$-Orbital conjugation and rehybridization in bridged annulenes and deformed molecules in general: $\pi$-orbital axis vector analysis. Pure and Applied Chemistry 1986;58(1):137-42.

[31] Palmer RE, Haddon RC, Kroto HW, Sermon PA. The Fullerenes: Powerful Carbon-Based Electron Acceptors: Discussion. Philosophical Transactions of the Royal Society of London Series A 1993;343:61-2. 
[32] Marsusi F, Qasemnazhand M. Sila-fulleranes: promising chemically active fullerene analogs. Nanotechnology 2016;27(27):275704.

[33] Casolo S, Løvvik OM, Martinazzo R, Tantardini GF. Understanding adsorption of hydrogen atoms on graphene. J Chem Phys 2009;130(5):054704.

[34] Yazyev OV, Helm L. Defect-induced magnetism in graphene. Phys Rev B 2007;75(12):125408.

[35] Šljivančanin Ž, Balog R, Hornekær L. Magnetism in graphene induced by hydrogen adsorbates. Chem Phys Lett 2012;541:70-74.

[36] Sofo JO, Suarez AM, Usaj G, Cornaglia PS, Hernández-Nieves AD, Balseiro CA. Electrical control of the chemical bonding of fluorine on graphene. Phys Rev B 2011;83(8):081411.

[37] Sadeghi A, Neek-Amal M, Berdiyorov GR, Peeters FM. Diffusion of fluorine on and between graphene layers. Phys Rev B 2015;91(1):014304.

[38] Santos EJ, Ayuela A, Sánchez-Portal D. Universal magnetic properties of sp $^{3}$-type defects in covalently functionalized graphene. New J of Phys 2012;14(4):043022.

[39] Kim HJ, Cho JH. Fluorine-induced local magnetic moment in graphene: A hybrid DFT study. Phys Rev B 2013;87(17):174435.

[40] Nair RR, Sepioni M, Tsai IL, Lehtinen O, Keinonen J, Krasheninnikov AV, Thomson T, Geim AK, Grigorieva IV. Spin-half paramagnetism in graphene induced by point defects. Nat Phys 2012;8(3):199.

[41] Gonze X, Beuken JM, Caracas R, Detraux F, Fuchs M, Rignanese GM, Sindic L, Verstraete M, Zerah G, Jollet F, Torrent M. First-principles computation of material properties: the ABINIT software project. Comput Mater Sci 2002;25(3):478-92.

[42] http://lammps.sandia.gov

[43] Van Duin AC, Dasgupta S, Lorant F, Goddard WA. ReaxFF: a reactive force field for hydrocarbons. J Phys Chem A 2001;105(41):9396-409.

[44] Singh SK, Srinivasan SG, Neek-Amal M, Costamagna S, Van Duin AC, Peeters FM. Thermal properties of fluorinated graphene. Phys Rev B 2013;87(10):104114.

[45] Singh SK. SK Singh, S. Costamagna, M. Neek-Amal, and FM Peeters. J Phys Chem C 2013;118:4460. 
[46] Himmetoglu B, Floris A, De Gironcoli S, Cococcioni M. Hubbard-corrected DFT energy functionals: The LDA+ U description of correlated systems. Int J Quant Chem 2014;114(1):1449.

[47] Giannozzi P, Baroni S, Bonini N, Calandra M, Car R, Cavazzoni C, Ceresoli D, Chiarotti GL, Cococcioni M, Dabo I, Dal Corso A. QUANTUM ESPRESSO: a modular and open-source software project for quantum simulations of materials. J Phys: Condensed Matt 2009;21(39):395502.

[48] Troullier N, Martins JL. Efficient pseudopotentials for plane-wave calculations. Phys Rev B 1991;43(3):1993.

[49] Perdew JP, Burke K, Ernzerhof M. Generalized gradient approximation made simple. Phys Rev Lett 1996;77(18):3865.

[50] Monkhorst HJ, Pack JD. Special points for Brillouin-zone integrations. Phys Rev B 1976;13(12):5188.

[51] Sofo JO, Chaudhari AS, Barber GD. Graphane: A two-dimensional hydrocarbon. Phys Rev B 2007;75(15):153401.

[52] Grimme S. Semiempirical GGA-type density functional constructed with a long-range dispersion correction. Journal of computational chemistry 2006;27(15):1787-99.

[53] Jónsson H, Mills G, Jacobsen KW. Nudged elastic band method for finding minimum energy paths of transitions. In Classical and quantum dynamics in condensed phase simulations 1998 (pp. 385-404).

[54] Wehling TO, Katsnelson MI, Lichtenstein AI. Impurities on graphene: Midgap states and migration barriers. Phys Rev B 2009;80(8):085428.

[55] Löwdin PO. Quantum theory of many-particle systems. I. Physical interpretations by means of density matrices, natural spin-orbitals, and convergence problems in the method of configurational interaction. Phys Rev 1955;97(6):1474.

[56] Makov G, Payne MC. Periodic boundary conditions in ab initio calculations. Phys Rev B 1995;51(7):4014.

[57] National Standard Reference Data Series. National Bureau of Standards, No. 31, Washington, DC, 1970; Benson SW. III-Bond energies. J Chem Edu 1965;42(9):502.

[58] Nakajima T, editor. Fluorine-carbon and fluoride-carbon materials: chemistry, physics, and applications. CRC Press; 2001. 
[59] Nakajima T, Groult H, editors. Fluorinated materials for energy conversion. Elsevier; 2005.

[60] Rudenko AN, Keil FJ, Katsnelson MI, Lichtenstein AI. Adsorption of diatomic halogen molecules on graphene: a van der Waals density functional study. Phys Rev B. 2010;82(3):035427.

[61] Yang Y, Liu FC, Kawazoe Y. Adsorption and diffusion of $\mathrm{F}_{2}$ molecules on pristine graphene. Chinese Physics B 2018;27(10):106801.

[62] Casolo S, Flage-Larsen E, Løvvik OM, Darling GR, Tantardini GF. Role of the selfinteraction error in studying chemisorption on graphene from first-principles. Phys Rev B 2010;81(20):205412.

[63] Ruiz E, Salahub DR, Vela A. Charge-transfer complexes: Stringent tests for widely used density functionals. J Phys Chem 1996;100(30):12265-76.

[64] A, Perdew JP, Csonka GI, Vydrov OA, Scuseria GE. Spurious fractional charge on dissociated atoms: Pervasive and resilient self-interaction error of common density functionals. J Chem Phys 2006;125(19):194112.

[65] Marsusi F, Verstraete MJ. Localization of electrons and magnetization in fluoro-graphene: A DFT+ U study. arXiv preprint arXiv:1709.09363.2017 Sep 27.

[66] Lieb EH. Two theorems on the Hubbard model. Phys Rev Lett 1989;62(10):1201.

[67] Zhou J, Liang Q, Dong J. Enhanced spin-orbit coupling in hydrogenated and fluorinated graphene. Carbon 2010;48(5):1405-9. 